\title{
Distributed energy-aware resource allocation in multi-antenna multi-carrier interference networks with statistical CSI
}

\author{
Alessio Zappone ${ }^{1 *}$, Giuseppa Alfano ${ }^{2}$, Stefano Buzzi ${ }^{3}$ and Michela Meo ${ }^{2}$
}

\begin{abstract}
Resource allocation for energy efficiency optimization in multi-carrier interference networks with multiple receive antennas is tackled. First, a one-hop network is considered, and then, the results are extended to the case of a two-hop network in which amplify-and-forward relaying is employed to enable communication. A distributed algorithm which optimizes a system-wide energy-efficient performance function, and which is guaranteed to converge to a stable equilibrium point, is provided. Unlike most previous works, in the definition of the energy efficiency, not only the users' transmit power but also the circuit power that is required to operate the devices is taken into account. All of the proposed procedures are guaranteed to converge and only require statistical channel state information, thus lending themselves to a distributed implementation. The asymptotic regime of a saturated network in which both the active users and the number of receive antennas deployed in each receiver grow large is also analyzed. Numerical results are provided to confirm the merits of the proposed algorithms.
\end{abstract}

\section{Introduction}

In the recent past, the growing concerns on sustainable growth due to the exponential increase in the use of mobile communication devices, as well as the need to maximize battery life in mobile handsets, garnered a great interest for resource allocation techniques aimed at the maximization of the energy efficiency (EE), measured in bits per Joule, at the physical layer of wireless networks. While more traditional resource allocation techniques pursue the optimization of the network's performance in terms of throughput, which usually comes at the expense of battery life, an energy-efficient resource allocation aims at the maximization of performance measures that take into account the trade-off between achieving a high throughput and saving as much battery power as possible.

Pioneering works in the area of distributed resource allocation for EE are [1-3]. There, the EE is defined as the ratio between the users' throughput and the transmit

\footnotetext{
*Correspondence: alessio.zappone@tu-dresden.de

1 Dresden University of Technology, Communication Theory Laboratory, Dresden 01062, Germany

Full list of author information is available at the end of the article
}

power, and energy-efficient power control algorithms for the uplink of wireless networks are provided.

The results of [1-3] have been extended in several directions in many following studies. In [4], joint linear receiver design and power control is pursued for CDMA networks, while in [5], spreading code optimization is also plugged into the resource allocation process. In [6], the impact of widely linear filtering on the $\mathrm{EE}$ of wireless networks is investigated. In [7], a hierarchical approach based on Stackelberg games is used to tackle the energy-efficient power control problem, while in [8], a power control algorithm based on a repeated-game approach is proposed. In [9], the impact of relaying on the EE of wireless networks is studied.

Most of the above works deal with multiple-accesschannel systems. In [10], instead, a multi-carrier interference network is considered, and the problem of distributed EE maximization through individual power allocation over the available subcarriers is investigated. However, the proposed algorithm is not guaranteed to converge to a stable equilibrium point. In [11], the uplink of multi-cell orthogonal frequency-division multiple access (OFDMA) network is considered, and a resource allocator aiming at minimizing the total transmitted

\section{是 Springer}

(c) 2013 Zappone et al: licensee Springer. This is an Open Access article distributed under the terms of the Creative Commons Attribution License (http://creativecommons.org/licenses/by/2.0), which permits unrestricted use, distribution, and reproduction in any medium, provided the original work is properly cited. 
power subject to individual rate constraints is studied. The results indicate that the proposed algorithm converges to a stable resource allocation policy only when the interference load is below a certain threshold. In [12], a game-theoretic approach to subcarriers, modulation format, and power allocation for transmit power minimization with rate constraints is considered. Again, the proposed game is not guaranteed to converge to an equilibrium, and for this reason, a virtual referee is introduced to dictate the resource allocation and force it to a stable and efficient equilibrium point. In [13], an auction approach to subcarrier, modulation, and coding scheme allocation in single-cell and multi-cell OFDMA networks is proposed, and the simplification is made to divide the users in each cell between interior and edge users, assuming that the inner users in each cell do not interfere with adjacent cells. In [14], energy-efficient subcarrier and transmit power allocation in the uplink of an OFDMA interference network is considered. However, in order to ensure the convergence of the resulting algorithm, subcarrier assignment is performed in a centralized fashion. In [15], a novel system-wide energy-efficient performance metric is proposed, which allows for a completely distributed and convergent power and subcarrier allocation in multi-carrier interference networks. There, the resource allocation problem is formulated as a potential game [16], with potential function given by the systemwide performance function to be maximized. Potential games for resource allocation in wireless multi-user networks have also been considered in [17-19].

In [15], as in all previously cited works, as far as the definition of the EE is concerned, only the transmit power is considered, whereas a more meaningful definition of the EE is obtained when not only the transmit power but also the power that is dissipated in the electronic circuitry of each terminal in order to operate the device is accounted for [20-25]. Moreover, previous works on distributed EE in interference networks deal with singleantenna systems, whereas it is confirmed that a crucial key ingredient of today's and future wireless networks will be the use of multiple antennas, which may be very helpful in providing diversity gains and dramatically increasing the system performance in terms of effective data rates and network coverage [26-28]. First, results on EE in multiple-antenna systems are [22,29], where singleuser multiple-input multiple-output (MIMO) systems are considered and the optimal energy-efficient covariance matrix is determined.

Motivated by this background, this paper extends the results of [15] in the following directions:

- The transmitter circuit powers are taken into account in the definition of the users' energy efficiency, which complicates the analysis.
- Multiple-antenna receivers are considered, and unlike [15], only statistical channel state information (CSI) is assumed at the transmitter side, which reduces the amount of feedback required to implement the proposed algorithms.

- A two-hop interference network in which communication takes place by means of an amplify-and-forward relay is also considered.

In this challenging scenario, distributed algorithms for energy-efficient joint subcarrier and power allocation are devised. The proposed algorithms require only the solution of convex problems, which makes their implementation feasible in real world systems, and will be shown to always converge to a stable equilibrium point. EE maximization will also be tackled in the asymptotic regime of a saturated interference network in which both the number of active users and of receive antennas grow large with a fixed ratio.

The remainder of this paper is organized as follows. Section 2 describes the considered system model. Section 3 investigates the energy-efficient resource allocation problem, providing the tools to devise suitable resource allocation algorithms. Section 4 contains the asymptotic analysis for saturated networks. In Section 5, the results of 3 are extended to the relay-assisted scenario, while Section 6 is devoted to the numerical results. Concluding remarks are given in Section 7.

\section{System model}

Consider the uplink of a $K \times J$ multi-carrier interference network. Each receiver is equipped with $M$ receive antennas, and for all $k=1, \ldots, K, a_{k} \in\{1,2, \ldots, J\}$ denotes the intended receiver for user $k$. It is assumed here that receiver assignment has been performed in a previous phase, and we focus on the resource allocation problem only. Let $\mathcal{L}=\{1,2, \ldots, L\}$ be the set of available subcarriers in the system, and denote by $p_{k}(\ell)$ the $k$-th user's transmit power on subcarrier $\ell$, and by $h_{k, j, m}(\ell)$ the complex channel gain between the $k$-th user and the $m$-th antenna of the $j$-th receiver on the $\ell$-th subcarrier, modeled as a realization of a zero-mean Gaussian random variable with variance $\frac{d_{k, j}^{-\eta}}{M}$, with $d_{k, j}$ and $\eta$ denoting the distance between the $k$-th mobile user and the $j$-th BS, and the path loss exponent, respectively. Let us also define the channel vector $\boldsymbol{h}_{k, j}(\ell)=\left[h_{k, j, 1}(\ell), \ldots, h_{k, j, M}(\ell)\right]^{T}$, with $(\cdot)^{T}$ denoting transpose, modeled as a zero-mean Gaussian random vector with covariance matrix $\boldsymbol{R}_{k, j}(\ell)$.

In the following, only statistical CSI is assumed at the transmitters' side, in terms of second-order statistics of the channel coefficients and vectors.

Now, for all $j=1, \ldots, J$, the data received on the $M$ antennas over subcarrier $\ell$ can be expressed as the $M$-dimensional vector 


$$
\boldsymbol{r}_{j}(\ell)=\left[r_{j, 1}(\ell), \ldots, r_{j, M}(\ell)\right]^{T}=\sum_{k=1}^{K} \sqrt{p_{k}(\ell)} \boldsymbol{h}_{k, j}(\ell) b_{k}(\ell)+\boldsymbol{w}_{j}(\ell),
$$

wherein $b_{k}(\ell)$ is the information symbol transmitted by the $k$-th user on the $\ell$-th subcarrier frequency, $\boldsymbol{w}_{j}(\ell)=$ $\left[w_{j, 1}(\ell), \ldots, w_{j, M}(\ell)\right]^{T}$ is the additive noise term, modeled as a zero-mean Gaussian random vector with covariance matrix $\sigma^{2} I_{M}$. In each receiver, the received data are processed by means of maximum ratio combining (MRC). The resulting instantaneous signal-to-interference-plusnoise ratio (SINR) for the $k$-th user is thus expressed, for all $k=1, \ldots, K$, as

$$
\gamma_{k, a_{k}}^{i n s t}(\ell)=\frac{p_{k}(\ell)\left\|\boldsymbol{h}_{k, a_{k}}(\ell)\right\|^{4}}{\sigma^{2}\left\|\boldsymbol{h}_{k, a_{k}}(\ell)\right\|^{2}+\sum_{i \neq k} p_{i}(\ell)\left|\boldsymbol{h}_{k, a_{k}}^{H}(\ell) \boldsymbol{h}_{i, a_{k}}(\ell)\right|^{2}} .
$$

In this scenario, the aim is to devise a transmit power and subcarrier allocation algorithm that optimizes the network's EE in a distributed way. Here, by distributed, we mean that the resource allocation process is not jointly carried out by a computational center which computes the optimum resource allocation policy, feeding back the results to the transmitters. Instead, each transmitter should allocate its own resources in a self-organizing way. This is a very important feature especially in networks that, by their own nature, lack a central control unit that dictates the resource allocation policy, which is the case for example in relevant communication systems like $a d$ hoc networks and interference networks.

\section{Distributed energy-efficient resource allocation}

The aim of this section is to derive a distributed energyefficient resource allocation algorithm. The main challenge in deriving a distributed algorithm in interference networks is that typically global CSI is needed. Otherwise stated, each user also needs to know other users' channels, which requires a too great amount of overhead information to feedback. In order to circumvent this problem, the proposed algorithm will be designed so as to require only statistical CSI at the transmitter side. Since channel statistics vary at a very slow rate compared to the actual channel realizations, feeding back only statistical CSI significantly reduces the amount of required overhead. Moreover, channel statistics can be estimated more easily than channel coefficients at the receivers. It is also to be mentioned that present multi-cell networks are typically endowed with a high-speed backhaul link which allows the receivers to exchange information with one another. Therefore, each receiver can easily learn the channel statistics also of users that are not associated to it and then feedback this information to its associated transmitters. Again, the overhead information to be exchanged on the backhaul link due to the resource allocation algorithm is quite limited since only channel statistics need to be shared. Thanks to these features, the algorithm to be developed lends itself to a distributed implementation at the transmitter side.

To begin with, we remark that since the resource allocation takes place at the transmitters, the instantaneous SINR expression (2) cannot be used for resource allocation purposes, because each transmitter only has statistical CSI. Thus, before turning to the analysis of the resource allocation algorithm, an average SINR expression is needed.

\subsection{Users' average SINR}

The average power $U_{k, a_{k}}(\ell)$ of the $k$-th user's intended symbol, in his assigned receiver $a_{k}$, on subcarrier $\ell$, is given by

$$
\begin{aligned}
U_{k, a_{k}}(\ell) & =p_{k}(\ell) E\left[\left|\boldsymbol{h}_{k, a_{k}}^{H}(\ell) \boldsymbol{h}_{k, a_{k}}(\ell)\right|^{2}\right] \\
& =p_{k}(\ell) E\left[\left|\boldsymbol{\alpha}_{k, a_{k}}^{H}(\ell) \boldsymbol{R}_{k, a_{k}}(\ell) \boldsymbol{\alpha}_{k, a_{k}}(\ell)\right|^{2}\right],
\end{aligned}
$$

with $\boldsymbol{\alpha}_{k, a_{k}}(\ell)=\boldsymbol{R}_{k, a_{k}}^{-1 / 2}(\ell) \boldsymbol{h}_{k, a_{k}}(\ell)$. Elaborating, $U_{k, a_{k}}(\ell)$ can be expressed as

$$
\begin{aligned}
U_{k, a_{k}}(\ell)= & p_{k}(\ell) E\left[\left|\sum_{m=1}^{M} \sum_{v=1}^{M} R_{k, a_{k}, \ell}(m, v) \alpha_{k, a_{k}, \ell}(m) \alpha_{k, a_{k}, \ell}^{*}(v)\right|^{2}\right] \\
= & p_{k}(\ell) E\left[\sum_{m=1}^{M} R_{k, a_{k}, \ell}^{2}(m, m)\left|\alpha_{k, a_{k}, \ell}(m)\right|^{4}\right. \\
& +2 \sum_{m=1}^{M} \sum_{v=m+1}^{M} R_{k, a_{k}, \ell}(m, m) R_{k, a_{k}, \ell}(v, v)\left|\alpha_{k, a_{k}, \ell}(m)\right|^{2}\left|\alpha_{k, a_{k}, \ell}(v)\right|^{2} \\
& \left.+4 \sum_{m=1}^{M} \sum_{v=m+1}^{M}\left|R_{k, a_{k}, \ell}(m, v)\right|^{2}\left|\alpha_{k, a_{k}, \ell}(m)\right|^{2}\left|\alpha_{k, a_{k}, \ell}(v)\right|^{2}\right] \\
= & p_{k}(\ell)\left[\sum_{m=1}^{M} R_{k, a_{k}, \ell}^{2}(m, m)+4 \sum_{m=1}^{M} \sum_{v=m+1}^{M}\left|R_{k, a_{k}, \ell}(m, v)\right|^{2}\right. \\
& \left.+2 \sum_{m=1}^{M} R_{k, a_{k}, \ell}^{2}(m, m)+2 \sum_{m=1}^{M} \sum_{v=m+1}^{M} R_{k, a_{k}, \ell}(m, m) R_{k, a_{k}, \ell}(v, v)\right] \\
= & p_{k}(\ell)\left(\operatorname{tr}^{2}\left(\boldsymbol{R}_{k, a_{k}}(\ell)\right)+2 \operatorname{tr}\left(\boldsymbol{R}_{k, a_{k}}(\ell) \boldsymbol{R}_{k, a_{k}}^{H}(\ell)\right)\right),
\end{aligned}
$$

wherein we have exploited the fact that $E\left[\left|\alpha_{k, a_{k}, \ell}(m)\right|^{4}\right]=$ 3 for all $m=1, \ldots, M$, because $\alpha_{k, a_{k}, \ell}(m)$ is a standard Gaussian variable for all $m=1, \ldots, M$. As for the average power of the interference-plus-noise term, we have

$$
\begin{aligned}
I_{k, a_{k}}(\ell)= & \sigma^{2} E\left[\left\|\boldsymbol{h}_{k, a_{k}}(\ell)\right\|^{2}\right] \\
& +\sum_{i \neq k} p_{i}(\ell) \operatorname{tr}\left(E\left[\boldsymbol{h}_{i, a_{k}}(\ell) \boldsymbol{h}_{i, a_{k}}^{H}(\ell) \boldsymbol{h}_{k, a_{k}}(\ell) \boldsymbol{h}_{k, a_{k}}^{H}(\ell)\right]\right) \\
= & \sigma^{2} \operatorname{tr}\left(\boldsymbol{R}_{k, a_{k}}(\ell)\right)+\sum_{i \neq k} p_{i}(\ell) \operatorname{tr}\left(\boldsymbol{R}_{i, a_{k}}(\ell) \boldsymbol{R}_{k, a_{k}}(\ell)\right),
\end{aligned}
$$


where the last equality stems from the assumption that the vectors $\boldsymbol{h}_{i, a_{k}}(\ell)$ and $\boldsymbol{h}_{k, a_{k}}(\ell)$ are statistically independent for all $k \neq i$. Finally, the $k$-th user's average SINR, in his intended receiver, on subcarrier $\ell$, is computed as

$$
\begin{aligned}
\gamma_{k, a_{k}}(\ell) & =\frac{U_{k, a_{k}}(\ell)}{I_{k, a_{k}}(\ell)} \\
& =\frac{p_{k}(\ell)\left(\left(\operatorname{tr}^{2}\left(\boldsymbol{R}_{k, a_{k}}(\ell)\right)+2 \operatorname{tr}\left(\boldsymbol{R}_{k, a_{k}}(\ell) \boldsymbol{R}_{k, a_{k}}^{H}(\ell)\right)\right)\right.}{\sigma^{2} \operatorname{tr}\left(\boldsymbol{R}_{k, a_{k}}(\ell)\right)+\sum_{i \neq k} p_{i}(\ell) \operatorname{tr}\left(\boldsymbol{R}_{i, a_{k}}(\ell) \boldsymbol{R}_{k, a_{k}}(\ell)\right)} .
\end{aligned}
$$

\subsection{Proposed distributed algorithm}

Having derived an average expression for the users' SINR, we are ready to start our analysis of the resource allocation problem. Mathematically, the distributed energy-efficient resource allocation problem is formulated as the $K$ coupled problems

$$
\begin{array}{ll}
\max _{\left\{p_{k}(\ell)\right\}_{\ell=1}^{L}} G\left(\left\{p_{k}(\ell), \gamma_{k}(\ell)\right\}_{k=1, \ell=1}^{K, L}\right) & \forall k=1, \ldots, K \\
\text { s.t. } \quad p_{k}(\ell) \geq 0 \quad \forall \ell=1, \ldots, L, \sum_{\ell=1}^{L} p_{k}(\ell) & \\
& \leq P_{\max , k}
\end{array}
$$

with $G\left(\left\{p_{k}(\ell), \gamma_{k}(\ell)\right\}_{k=1, \ell=1}^{K, L}\right)$ being the energy-efficient performance metric to optimize, which will be specified shortly. For all $k=1, \ldots, K$, the solution to the $k$-th problem in (6) yields the $k$-th user's power allocation for a fixed configuration of the other users' powers, and any fixed point of iteration (6) represents a stable resource allocation policy. We remark that for all $k=1, \ldots, K$, only the transmit powers $\left\{p_{k}(\ell)\right\}_{\ell=1}^{L}$ have been indicated as the optimization variables of the generic $k$-th problem, because by choosing the transmit powers, each user automatically chooses also the transmit subcarriers. Indeed, a subcarrier can be discarded by simply transmitting zero power over it.

Now, traditionally, the EE of a single communication link is defined as the ratio between the achieved throughput and the transmitted power, which can be mathematically expressed as $[4,10,30]$, and references therein

$$
\mathrm{EE}^{p c}=R \frac{D}{Q} \frac{\left(1-e^{-\gamma}\right)^{Q}}{p},
$$

wherein $R$ is the transmit data rate, $Q \geq 1$ is the packet length, $D \leq Q$ is the number of information symbols contained in each packet, $\gamma$ is the achieved SINR, $p$ the transmit power, and $\left(1-e^{-\gamma}\right)^{Q}$ is the so-called efficiency function which approximates the probability of correct reception for a data-packet of length $Q[4,10,30]$ and references therein. We stress that the case of bit-oriented communications, i.e., $Q=1$ is included as a special case in our definition of the energy efficiency and all results to follow will hold true also for $Q=1$. Moreover, it should be mentioned that also the case $Q>1$ is of practical interest in modern OFDMA systems, such as LTE [31].

Another widely used efficiency function is the achievable rate $\log (1+\gamma)[21,23,32]$. However, such a choice applies to strictly static channels but has no informationtheoretic meaning in the considered scenario where the channels are rapidly varying. In our context, an information-theoretic meaningful function would be the ergodic achievable rate $E[\log (1+\gamma)]$. Such an approach, which has been considered in [22] for the simpler scenario of single-user MIMO systems, appears more challenging in interference networks and is left as future work.

In the considered multi-carrier system, for all $k=$ $1, \ldots, K,(7)$ can be seen as the per-carrier EE of a given user. Then, recalling that each user is assigned $L$ subcarriers, the EE with which the generic $n$-th user transmits each packet of $Q$ bits is given by [10]

$$
\mathrm{EE}_{n}=R \frac{D}{Q} \frac{\sum_{\ell=1}^{L}\left(1-e^{-\gamma_{n, a_{n}}(\ell)}\right)^{Q}}{\sum_{\ell=1}^{L} p_{n}(\ell)+P_{c, n}}
$$

while the network's global energy efficiency (GEE), defined as the ratio of network's global throughput over the network's total consumed power, is written as

$$
\mathrm{GEE}=\frac{\sum_{n=1}^{K} R \frac{D}{Q} \sum_{\ell=1}^{L}\left(1-e^{-\gamma_{n, a_{n}}(\ell)}\right)^{Q}}{\sum_{n=1}^{K} \sum_{\ell=1}^{L} P_{c, n}+p_{n}(\ell)},
$$

wherein the circuit power $P_{c, n}$ that is needed to operate transmitter $n$ has also been included in the expression of the consumed power. From a user-centric point of view, individual maximization of (8), for all $n=1, \ldots, K$ should be pursued. However, this would result in iterations (6) to be not always convergent, and even if convergence occurs, the resulting power allocation policy may not be efficient from a social welfare point of view. Indeed, from a social welfare point of view, the GEE (9) would be a canonical choice as the objective $G\left(\left\{p_{k}(\ell), \gamma_{k}(\ell)\right\}_{k=1, \ell=1}^{K, L}\right)$ of (6). However, the drawback of maximizing of (9) is that it might lead to unfair power allocations. Indeed, due to its additive nature, the maximum of (9) might be obtained by having users with very low channel coefficients transmit at very low powers. In order to compromise between the need to achieve improved overall EE and the need to obtain a fair resource allocation, similarly to [15], the following multiplicative version of (9) will be considered as objective of (6), namely

$$
\widetilde{\mathrm{GEE}}=\frac{\prod_{n=1}^{K} R \frac{D}{Q} \prod_{\ell=1}^{L}\left(1-e^{-\gamma_{n, a_{n}}(\ell)}\right)^{Q}}{\prod_{n=1}^{K} \prod_{\ell=1}^{L}\left(p_{n}(\ell)+P_{c, n}\right)} .
$$


Due to its multiplicative nature, it is unlikely that a maximizer of (10) results in one of the users' throughputs to be very low, since each user's throughputs is a factor of the product in the numerator of (10). Moreover, (10) is also a system-wide performance function, since it is an increasing function of the players' energy efficiencies. We stress that the maximization of products of utility functions in order to obtain fair resource allocation policies is also considered in contexts other than EE maximization [33,34].

However, the drawback of this approach is that the maximization of (10) is clearly more complex than that of (9). Thus, in order to obtain a mathematically treatable objective function, the traditional efficiency function $\left(1-e^{-\gamma}\right)^{Q}$ will be approximated by $\left(e^{-\beta_{n} / \gamma}\right)^{Q}$, with $\beta_{n}$ a suitable constant to be specified. Note that, similarly to $\left(1-e^{-\gamma}\right)^{Q}$, the modified efficiency function $\left(e^{-\beta_{n} / \gamma}\right)^{Q}$ is still an $\mathrm{S}$ shaped $^{\mathrm{a}}$, increasing function of $\gamma$, approaching zero for $\gamma \rightarrow 0$ and approaching unity for $\gamma \rightarrow+\infty$. Aiming at approximating $\left(1-e^{-\gamma}\right)^{Q}$ with $\left(e^{-\beta_{n} / \gamma}\right)^{Q}$, a natural choice for $\beta_{n}$ is to set it so as to minimize the mean square error between the two functions. Accordingly, for all $n=$ $1, \ldots, K, \beta_{n}$ is determined as the solution to the problem

$$
\left.\min _{\beta_{n}} \int_{0}^{\gamma_{\max , n}}\left[\left(1-e^{-x}\right)\right)^{Q}-e^{-Q \beta_{n} / x}\right]^{2} d x
$$

wherein $\gamma_{\max , n}$ is the maximum SINR that user $n$ can attain. For the case at hand, we have

$$
\gamma_{\max , n}=\frac{P_{\max , n} E\left[\left\|\boldsymbol{h}_{n, a_{n}}(\ell)\right\|^{2}\right]}{\sigma^{2}}
$$

i.e., the $n$-th user's SINR with interference-free transmission. The following proposition holds.

Proposition 1. The solution to problem (11) is obtained as the solution to the equation

$$
\sum_{m=0}^{Q}\left(\begin{array}{l}
Q \\
m
\end{array}\right)(-1)^{m} \int_{0}^{\gamma_{\max , n}} \frac{e^{-m x}}{x} e^{-Q \beta_{n} / x}=-\mathrm{Ei}\left(-\frac{2 Q \beta_{n}}{\gamma_{\max , n}}\right)
$$

with $\mathrm{Ei}(\cdot)$ denoting the exponential integral function.

Proof. Setting the first-order derivative of the objective of (11) to zero yields

$$
\int_{0}^{\gamma_{\max , n}} \frac{\left(1-e^{-x}\right)^{Q} e^{-\frac{Q \beta_{n}}{x}}}{x} d x=\int_{0}^{\gamma_{\max , n}} \frac{e^{-2 \frac{Q \beta_{n}}{x}}}{x} d x .
$$

Applying Newton's binomial formula to the left-handside of (14) yields the left-hand-side of (13). Next, operating the substitution $y=\frac{2 Q \beta_{n}}{x}$ in the right-hand-side of (14) yields

$$
\int_{\frac{2 Q \beta_{n}}{\gamma_{\max , n}}}^{\infty} \frac{e^{-y}}{y} d y=-\mathrm{Ei}\left(-\frac{2 Q \beta_{n}}{\gamma_{\max , n}}\right) .
$$

Hence, the thesis.

It should be stressed that the computation of the coefficients $\left\{\beta_{n}\right\}_{k=1}^{K}$ needs to be performed just once and can be carried out off-line because each $\beta_{n}$ only depends on the constant networks parameters $Q, P_{\max , n}, \sigma^{2}$, and $E\left[\left\|\boldsymbol{h}_{n, a_{n}}(\ell)\right\|^{2}\right.$.

In order to further motivate the validity of the modified efficiency function as a substitute for the classical one, let us consider the ratio $\frac{e^{-\beta_{n} / \gamma}}{1-e^{-\gamma}}$. Note that for increasing $\gamma$, it converges to one. Moreover, in order to give an insight as to how large $\gamma$ is required to be for $\frac{e^{-\beta_{n} / \gamma}}{1-e^{-\gamma}}$ to approach unity, Figure 1 reports such a ratio for the case $\gamma_{\max , n}=100$. It is seen that for $\gamma>0 \mathrm{~dB}$ (that is the region of interest) $\frac{e^{-\beta_{n} / \gamma}}{1-e^{-\gamma}}$ is very close to 1 .

Thus, (10) can be approximated as

$$
\widehat{\mathrm{GEE}}=\frac{\prod_{n=1}^{K} R \frac{D}{Q} \prod_{\ell=1}^{L} e^{-\frac{Q \beta_{n}}{\gamma_{n, a_{n}}(\ell)}}}{\prod_{n=1}^{K} \prod_{\ell=1}^{L}\left(p_{n}(\ell)+P_{c, n}\right)},
$$

and the $k$-th problem in (6) can be restated as

$$
\left\{\begin{array}{l}
\max _{\left\{p_{k}(\ell)\right\}_{\ell=1}^{L}} \frac{\prod_{n=1}^{K} R \frac{D}{Q} \prod_{\ell=1}^{L}\left(e^{-Q \beta_{n} / \gamma_{n, a_{n}}(\ell)}\right)}{\prod_{n=1}^{K} \prod_{\ell=1}^{L}\left(p_{n}(\ell)+P_{c, n}\right)} \\
\text { s.t. } \quad p_{k}(\ell) \geq 0 \quad \forall \ell=1, \ldots, L, \sum_{\ell=1}^{L} p_{k}(\ell) \leq P_{\max , k}
\end{array} .\right.
$$

Accordingly, the resource allocation algorithm can be expressed as follows:

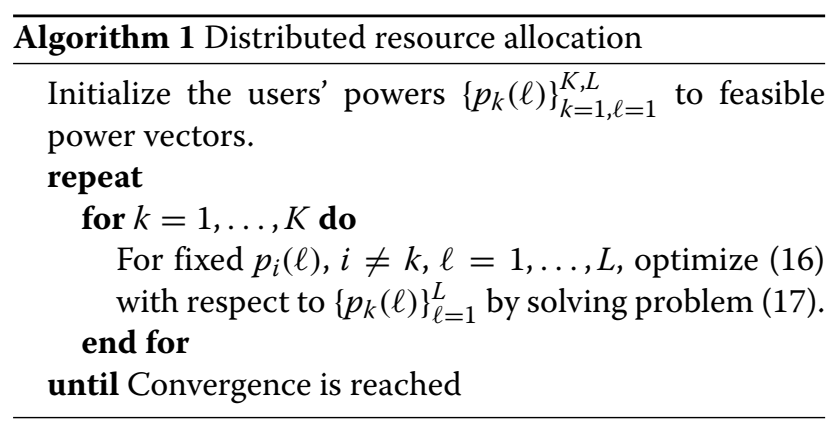

Convergence in Algorithm 1 is declared when the difference between the values of the objective function (16) achieved at the end of two successive outer loops is below a predetermined tolerance. The following proposition guarantees the convergence of Algorithm 1. 


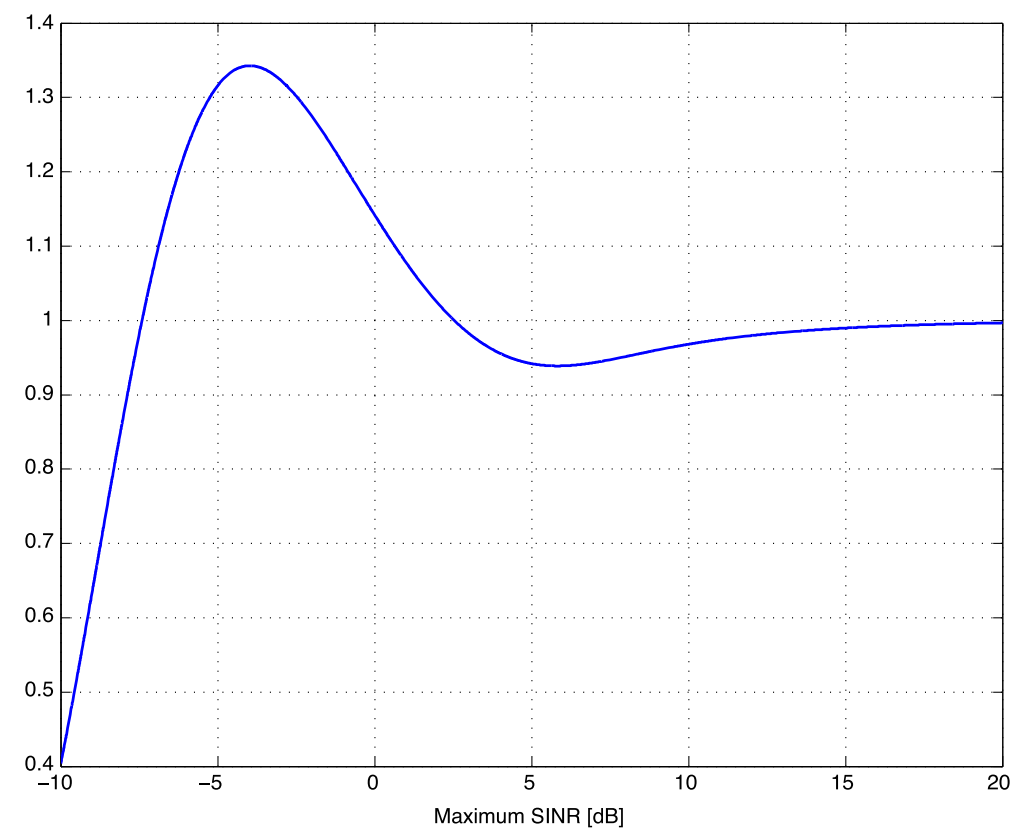

Figure 1 The quantity $\frac{e^{-\beta / \gamma}}{1-e^{-\gamma}}$ is plotted here versus $\gamma$ for the case $\gamma_{\max }=100$.

Proposition 2. For any feasible initialization point $\left\{p_{k}^{(0)}(\ell)\right\}_{k=1, \ell=1}^{K, L}$, Algorithm 1 is guaranteed to converge.

Proof. The objective (16) depends on all of the users' $K L$ transmit powers. After the initialization, we have $\widehat{\mathrm{GEE}}^{(0)}\left(\left\{p_{1}^{(0)}(\ell)\right\}_{\ell=1}^{L}, \ldots,\left\{p_{K}^{(0)}(\ell)\right\}_{\ell=1}^{L}\right)$. After the first iteration of the for cycle in Algorithm 1, (16) is maximized with respect to $\left\{p_{1}(\ell)\right\}_{\ell=1}^{L}$ while keeping the other $(K-1) L$ powers fixed. Let us denote by $\left\{p_{1}^{(1)}(\ell)\right\}_{\ell=1}^{L}$ the $L$ powers resulting from such optimization. Then, after this first optimization, the new value of the objective is $\widehat{\operatorname{GEE}}^{(1)}\left(\left\{p_{1}^{1}(\ell)\right\}_{\ell=1}^{L},\left\{p_{2}^{(0)}(\ell)\right\}_{\ell=1}^{L}, \ldots,\left\{p_{K}^{(0)}(\ell)\right\}_{\ell=1}^{L}\right)$, and clearly, we have $\widehat{\mathrm{GEE}}^{(1)} \geq \widehat{\mathrm{GEE}}^{(0)}$. In the second iteration of the cycle, the powers $\left\{p_{2}(\ell)\right\}_{\ell=1}^{L}$ are optimized. Thus, after the optimization, we have $\widehat{\operatorname{GEE}}^{(2)}\left(\left\{p_{1}^{1}(\ell)\right\}_{\ell=1}^{L},\left\{p_{2}^{(1)}(\ell)\right\}_{\ell=1}^{L},\left\{p_{3}^{(0)}(\ell)\right\}_{\ell=1}^{L}, \ldots\right.$, $\left.\left\{p_{K}^{(0)}(\ell)\right\}_{\ell=1}^{L}\right)$, and it holds $\widehat{\mathrm{GEE}}^{(2)} \geq \widehat{\mathrm{GEE}}^{(1)}$. It is seen that cyclically iterating this procedure originates a sequence of values of $\widehat{\mathrm{GEE}}^{(n)}$ which is non-decreasing. As a consequence, since $\widehat{\mathrm{GEE}}$ is upper-bounded with respect to the transmit powers, Algorithm 1 will eventually converge.

Now, in order to complete the resource allocation design, the solution to problem (17) remains to be tackled. Such a problem is not convex, because the objective is not concave, but it is possible to recast it as a convex problem without loss of optimality, by exploiting the result in the following proposition. Next, we will also provide an algorithm to solve problem (17) that employs the alternating maximization technique [35], rather than using the convex reformulation.

Proposition 3. For any $k=1, \ldots, K$, problem (17) can be restated as

$$
\left\{\begin{array}{l}
\max _{\left\{p_{k}(\ell)\right\}_{\ell=1}^{L}}-\sum_{\ell=1}^{L}\left(\frac{a_{k}(\ell)}{p_{k}(\ell)}+c_{k}(\ell) p_{k}(\ell)+\log \left(p_{k}(\ell)+P_{c, k}\right)\right) \\
\text { s.t. } \quad p_{k}(\ell) \geq 0 \quad \forall \ell=1, \ldots, L, \sum_{\ell=1}^{L} p_{k}(\ell) \leq P_{\max , k}
\end{array},\right.
$$

wherein

$$
a_{k}(\ell)=Q \beta_{k} \frac{\sigma^{2} \operatorname{tr}\left(\boldsymbol{R}_{k, a_{k}}(\ell)\right)+\sum_{i \neq k} p_{i}(\ell) \operatorname{tr}\left(\boldsymbol{R}_{i, a_{k}}(\ell) \boldsymbol{R}_{k, a_{k}}(\ell)\right)}{\left(\operatorname{tr}^{2}\left(\boldsymbol{R}_{k, a_{k}}(\ell)\right)+2 \operatorname{tr}\left(\boldsymbol{R}_{k, a_{k}}(\ell) \boldsymbol{R}_{k, a_{k}}^{H}(\ell)\right)\right)}
$$

$$
c_{k}(\ell)=\sum_{n \neq k} \frac{Q \beta_{n} \operatorname{tr}\left(\boldsymbol{R}_{k, a_{n}}(\ell) \boldsymbol{R}_{n, a_{n}}(\ell)\right)}{p_{n}(\ell)\left(\operatorname{tr}^{2}\left(\boldsymbol{R}_{n, a_{n}}(\ell)\right)+2 \operatorname{tr}\left(\boldsymbol{R}_{n, a_{n}}(\ell) \boldsymbol{R}_{n, a_{n}}^{H}(\ell)\right)\right)} .
$$

Moreover, the objective of (18) has a unique maximizer, which lies in its concave region. 
Proof. We start by observing that it is possible to apply any increasing function to (16) without changing its maximizers. Then, applying the logarithmic function yields

$$
\begin{aligned}
K \ln \left(\frac{R D}{Q}\right) & +\ln \left(\prod_{n=1}^{K} \prod_{\ell=1}^{L} \frac{\left(e^{-Q \beta_{n} / \gamma_{n, a_{n}}(\ell)}\right)}{\left(p_{n}(\ell)+P_{c, n}\right)}\right)=K \ln \left(\frac{R D}{Q}\right) \\
& -\sum_{n=1}^{K} \sum_{\ell=1}^{L}\left(\frac{Q \beta_{n}}{\gamma_{n, a_{n}}(\ell)}+\ln \left(p_{n}(\ell)+P_{c, n}\right)\right) .
\end{aligned}
$$

Then, plugging the expression for the users' SINRs and highlighting the terms that depend on the $k$-th user's transmit powers, (21) can be rewritten as

$$
\begin{aligned}
& -Q \beta_{k} \sum_{\ell=1}^{L} \frac{\sigma^{2} \operatorname{tr}\left(\boldsymbol{R}_{k, a_{k}}(\ell)\right)+\sum_{i \neq k} p_{i}(\ell) \operatorname{tr}\left(\boldsymbol{R}_{i, a_{k}}(\ell) \boldsymbol{R}_{k, a_{k}}(\ell)\right)}{p_{k}(\ell)\left(\left(\operatorname{tr}^{2}\left(\boldsymbol{R}_{k, a_{k}}(\ell)\right)+2 \operatorname{tr}\left(\boldsymbol{R}_{k, a_{k}}(\ell) \boldsymbol{R}_{k, a_{k}}^{H}(\ell)\right)\right)\right.} \\
& -\sum_{n \neq k} \sum_{\ell=1}^{L} \frac{Q \beta_{n} p_{k}(\ell) \operatorname{tr}\left(\boldsymbol{R}_{k, a_{n}}(\ell) \boldsymbol{R}_{n, a_{n}}(\ell)\right)}{p_{n}(\ell)\left(\left(\operatorname{tr}^{2}\left(\boldsymbol{R}_{n, a_{n}}(\ell)\right)+2 \operatorname{tr}\left(\boldsymbol{R}_{n, a_{n}}(\ell) \boldsymbol{R}_{n, a_{n}}^{H}(\ell)\right)\right)\right.} \\
& -\sum_{\ell=1}^{L} \ln \left(p_{k}(\ell)+P_{c, k}\right)+C_{k},
\end{aligned}
$$

with $C_{k}$ including all the terms that do not depend on the $k$-th user's transmit powers. Then, by inspection, it is seen that (56) coincides with the objective of (18), and the first part of the thesis is proved. In order to prove the second part, let us first note that (18) is not a convex problem, because its objective is not concave, as can be verified by direct computation of its Hessian. However, the objective of (18) has a unique maximum which lies within the concave region of the objective function. To prove this, let us denote each summand of the objective of (18) as the function

$$
g_{k}\left(p_{k}(\ell)\right)=-\left(\frac{a_{k}(\ell)}{p_{k}(\ell)}+c_{k}(\ell) p_{k}(\ell)+\log \left(p_{k}(\ell)+P_{c, k}\right)\right) .
$$

For all $k=1, \ldots, K$, the first derivative of $g_{k}\left(p_{k}(\ell)\right)$ is given by

$$
\frac{\partial g_{k}\left(p_{k}(\ell)\right)}{\partial p_{k}(\ell)}=\frac{a_{k}(\ell)}{p_{k}^{2}(\ell)}-c_{k}(\ell)-\frac{1}{p_{k}(\ell)+P_{c, k}} .
$$

Setting (24) to zero and elaborating yields the equation

$$
c_{k}(\ell) p_{k}^{3}(\ell)+\left(c_{k}(\ell) P_{c, k}+1\right) p_{k}^{2}(\ell)=a_{k}(\ell) p_{k}(\ell)+a_{k}(\ell) P_{c, k}
$$

which is guaranteed to admit a unique solution for positive $p_{k}(\ell)$, because an increasing cubic curve with zero intercept is on the left-hand-side, while a line with positive slope and intercept is on the right-hand-side. Denoting by $p_{k}^{*}(\ell)$ such a solution, it is also seen that, for all $k=$ $1, \ldots, K, g_{k}\left(p_{k}(\ell)\right)$ is an increasing function for $p_{k}(\ell) \leq$ $p_{k}^{*}(\ell)$, thus implying that $p_{k}^{*}(\ell)$ is a maximizer for $g_{k}(\ell)$. Hence, for all $k=1, \ldots, K$, it holds that

$$
\max \left(\sum_{\ell=1}^{L} g_{k}\left(p_{k}(\ell)\right)\right) \leq \sum_{\ell=1}^{L} \max \left(g_{k}\left(p_{k}(\ell)\right)\right)=\sum_{\ell=1}^{L} g_{k}\left(p_{k}^{*}(\ell)\right)
$$

which shows that $\left\{p_{k}^{*}(\ell)\right\}_{\ell=1}^{L}$ is the unique maximizer of the objective of (18). Next, let us compute the Hessian of the objective of (18). It is easy to realize that all the off-diagonal components equal zero, whereas for all $\ell=$ $1, \ldots, L$, we have

$$
\begin{aligned}
\frac{\partial^{2} u_{k}}{\partial p_{k}^{2}(\ell)} & =\frac{1}{\left(p_{k}(\ell)+P_{c, k}\right)^{2}}-2 \frac{a_{k}(\ell)}{p_{k}^{3}(\ell)} \\
& =\frac{p_{k}^{3}(\ell)-2 a_{k}(\ell)\left(p_{k}(\ell)+P_{c, k}\right)^{2}}{p_{k}^{3}(\ell)\left(p_{k}(\ell)+P_{c, k}\right)^{2}} .
\end{aligned}
$$

Thus, the resulting Hessian is a diagonal matrix with diagonal entries given by (27). Also, it is seen that (27) vanishes when $p_{k}^{3}(\ell)=2 a_{k}(\ell)\left(p_{k}(\ell)+P_{c, k}\right)^{2}$, which is guaranteed to admit a unique solution for positive $p_{k}(\ell)$ because the intersection between a positive cubic curve with zero intercept and a convex parabola with positive intercept is unique. For all $\ell=1, \ldots, L$, denote by $\bar{p}_{k}(\ell)$ such a solution. Then, by inspection, it can also be seen that (27) is negative for $p_{k}(\ell)<\bar{p}_{k}(\ell)$, thus implying that $g_{k}(\ell)$ is concave for $p_{k}(\ell)<\bar{p}_{k}(\ell)$. Consequently, $u_{k}=\sum_{\ell=1}^{L} g_{k}\left(p_{k}(\ell)\right)$ is concave when $p_{k}(\ell)<\bar{p}_{k}(\ell)$ for all $\ell=1, \ldots, L$. Now, in order to complete the proof, it is to be shown that $p_{k}^{*}(\ell) \leq \bar{p}_{k}(\ell)$, for all $\ell=1, \ldots, L$, which is equivalent to showing that the first-order derivative of $g_{k}(\ell)$ is negative when evaluated at $\bar{p}_{k}(\ell)$. To see this note that from the left-hand-side of (27), it follows that, for all $\ell=1, \ldots, L, \bar{p}_{k}(\ell)$ has to satisfy the equation $\frac{a_{k}(\ell)}{\bar{p}_{k}^{2}(\ell)}=\frac{\bar{p}_{k}(\ell)}{2\left(\bar{p}_{k}(\ell)+P_{c, k}\right)^{2}}$. Therefore, from (24), we have

$$
\begin{gathered}
\frac{\partial g_{k}\left(\bar{p}_{k}(\ell)\right)}{\partial p_{k}(\ell)}=\frac{a_{k}(\ell)}{\bar{p}_{k}^{2}(\ell)}-c_{k}(\ell)-\frac{1}{\bar{p}_{k}(\ell)+P_{c, k}}= \\
\frac{\bar{p}_{k}(\ell)}{2\left(\bar{p}_{k}(\ell)+P_{c, k}\right)^{2}}-c_{k}(\ell)-\frac{1}{\bar{p}_{k}(\ell)+P_{c, k}}= \\
-\frac{\bar{p}_{k}(\ell)+2 P_{c, k}+2 c_{k}(\ell)\left(\bar{p}_{k}(\ell)+P_{c, k}\right)}{2\left(\bar{p}_{k}(\ell)+P_{c, k}\right)}<0 .
\end{gathered}
$$

Hence, the thesis. 
Thus, Proposition 3 allows to reformulate the nonconvex problem (18) as a convex one by restricting the problem domain to the concave region of the objective function, which can be done by simply imposing the additional constraints $p_{k}(\ell) \leq \bar{p}_{k}(\ell)$ for all $\ell=1, \ldots, L$ in $(18)$. This causes no loss of optimality, since the global maximum has been proved to lie in the concave region of the objective function.

Moreover, since numerical algorithms might still be too complex when the network's load grows too large or in scenarios when computational complexity is a critical issue, in the following, we provide another, even more, computationally efficient technique to solve (18), based on the alternating maximization algorithm [35]. According to the alternating maximization, a function can be cyclically maximized with respect to one (or a block of) variable, while keeping the other variables fixed. For the case at hand, this means that in each cycle, problem (18) can be solved with respect to one of the transmit powers, say $p_{k}(\ell)$, while keeping the other powers $\left\{p_{k}(q)\right\}_{q \neq \ell}$ fixed, thus converting problem (18) into a sequence of scalar sub-problems, each of which can be solved in closedform. Indeed, when only the generic power $p_{k}(\ell)$ is to be optimized, problem (18) can be recast as

$$
\left\{\begin{array}{l}
\max _{p_{k}(\ell)} g_{k}\left(p_{k}(\ell)\right) \\
\text { s.t. } p_{k}(\ell) \geq 0, p_{k}(\ell) \leq P_{\max , k}-\sum_{q \neq \ell} p_{k}(q)
\end{array} .\right.
$$

In Proposition 2, it has already been proved that $g_{k}\left(p_{k}(\ell)\right)$ admits a unique maximizer $p_{k}^{*}(\ell)$ which is given by the solution to (25). Consequently, the solution to problem (30) is given by

$$
p_{k}(\ell)=\min \left\{\left(P_{\max , k}(\ell)-\sum_{q \neq \ell} p_{k}(q)\right), p_{k}^{*}(\ell)\right\} .
$$

Equipped with this result, the formal alternating maximization algorithm to solve problem (18) can be stated as follows
Similarly as for Algorithm 1, convergence in Algorithm 2 is declared when the value of the objective of (18) after two successive outer loops is below a given threshold. Convergence of Algorithm 2 can be proved with similar algorithms as for Algorithm 1.

From (31), it also follows that for large $P_{\max , k}$, Algorithm 2 is guaranteed to converge to the global solution of (18) in $L$ iterations. Indeed, for large $P_{\max , k}$, after $L$ iterations of the alternating maximization, the resulting transmit powers will be $p_{k}(\ell)=p_{k}^{*}(\ell)$, which is the global maximizer of solution of (18). In Section 6, the performance obtained using Algorithm 2 to solve (18) will be contrasted to that achieved by solving (18) through its convex reformulation.

\subsection{The proposed algorithm as a potential game}

In this section, we will briefly provide a different look on the proposed algorithm, showing how it fits into the framework of game theory and in particular of potential games. Let us first give some details on non-cooperative games and potential games.

In its strategic form, a game $\mathcal{G}$ can be described as a triplet $\mathcal{G}=\left\{\mathcal{K},\left\{\mathcal{S}_{k}\right\}_{k=1}^{K},\left\{u_{k}\right\}_{k=1}^{K}\right\}$, wherein $\mathcal{K}$ is the set of players (e.g., the communicating devices in a wireless network), $\mathcal{S}_{k}$ is the set of all possible strategies for the $k$-th player, and $u_{k}$ represents the utility function or payoff of the $k$-th player; $u_{k}$ is a scalar function depending on the strategies taken by all players of the game. Thus, a change in strategy from one player affects all the other players as well, and triggers a dynamic process, in which players iteratively update their own strategies as a reaction to changes in the strategies of the other players. This process is mathematically represented by the set of coupled problems

$$
\max _{s_{k}} u_{k}\left(s_{k}, s_{-k}\right), \quad \forall k \in \mathcal{K},
$$

with $s_{k}$ and $s_{-k}$ being the $k$-th player's strategy and set of the other player's strategies, respectively. The coupled problems (32) are usually referred to as best-response dynamics (BRD), because for all $k \in \mathcal{K}$, in the $k$-th iteration, given the strategies of the other players $\boldsymbol{s}_{-k}$, player $k$ responds by choosing his own strategy $s_{k}$ in order to

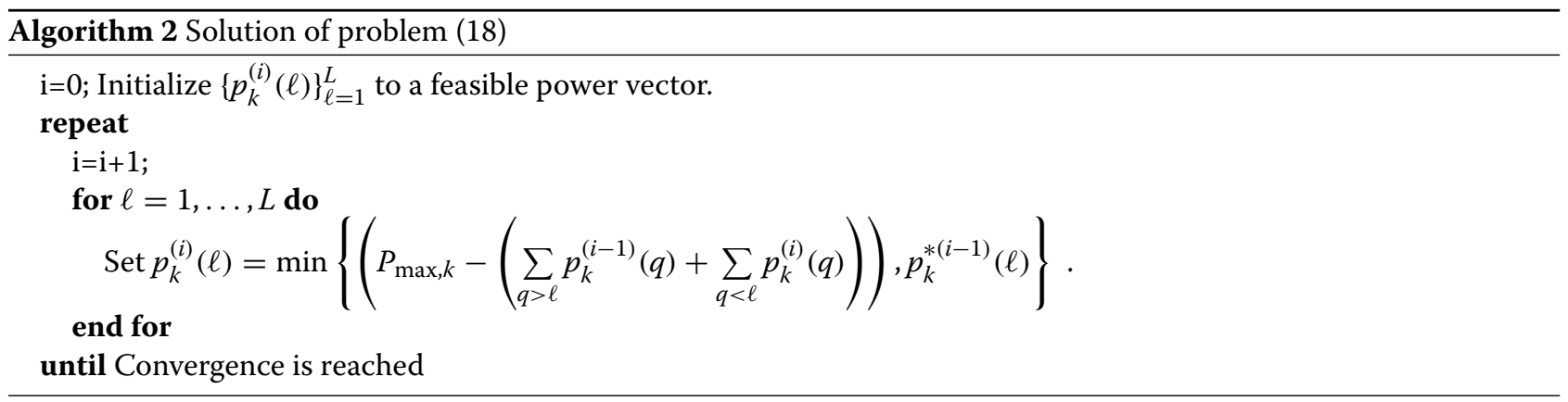


maximize his own utility function. Each fixed point of (32), if any, is termed Nash equilibrium (NE). At an NE, no user can unilaterally improve its own utility by taking a different strategy, thus implying that each user, provided that the other users' strategies do not change, is not interested in changing his own strategy. In general, given a generic strategic-form game, convergence of the BRD to an NE is not guaranteed, even if one or more NEs exist.

We give now the formal definition of a potential game [16]. A strategic game $\mathcal{G}=\left[\mathcal{K},\left\{\mathcal{S}_{k}\right\},\left\{u_{k}\right\}\right]$ is called an exact potential game if there exists a function $V: \mathcal{S}_{1} \times$ $\mathcal{S}_{2} \times \ldots \mathcal{S}_{K} \rightarrow \mathcal{R}$ such that for any $k \in \mathcal{K}$ and for any $\left(s_{k}, s_{-k}\right),\left(s_{k}^{*}, s_{-k}\right) \in \mathcal{S}_{1} y \times \mathcal{S}_{2} \times \ldots \mathcal{S}_{K}$, we have

$$
u_{k}\left(s_{k}, \boldsymbol{s}_{-k}\right)-u_{k}\left(s_{k}^{*}, \boldsymbol{s}_{-k}\right)=V\left(s_{k}, \boldsymbol{s}_{-k}\right)-V\left(s_{k}^{*}, \boldsymbol{s}_{-k}\right) .
$$

The function $V$ is called the potential function of the game. A very attractive property of potential games is that at least one NE is guaranteed to exist and that the BRD always converges to an NE, provided the potential function is upper-bounded. In our scenario, it can be seen that the distributed resource allocation algorithm can be seen as a potential game $\mathcal{G}_{\text {pot }}$, with the mobile users as players, with potential function $V$ given by (21) and utility functions given by $u_{k}=V-C_{k}$, for all $k=1, \ldots, K$, with $C_{k}$ being the additive constant that appears in (56). Thus, the resource allocation policy obtained at the fixed point of Algorithm 1 can be regarded as an $\mathrm{NE}$ of $\mathcal{G}_{\text {pot }}$.

\section{Energy-efficient resource allocation in saturated networks with fairness constraint}

In Section 3, an energy-efficient performance function has been proposed that results at the same time in improved overall network's performance and in a fair resource allocation to the users. This has been tackled assuming only partial CSI and thus operating with average users' SINR expressions. In this section, a different approach is taken. The scenario in which each user $k$ allocates his own resources to maximize the EE (8) of his own communication link is addressed. This problem will be tackled in the asymptotic regime of a saturated network in which the number of active users and the number of antennas equipped at each receiver grow to infinity with a fixed ratio $\frac{K}{M}=\alpha$. Unlike what happens in the non-asymptotic regime, it will be shown that in the asymptotic regime individual maximization of (8) is guaranteed to converge to an equilibrium. The problem can be formally stated as follows:

$$
\left\{\begin{array}{ll}
\max _{\left\{p_{k}(\ell) J_{\ell=1}^{L} R \frac{D}{Q} \frac{\sum_{\ell=1}^{L}\left(1-e^{-\gamma_{k, a_{k}}^{a s}(\ell)}\right)^{Q}}{\sum_{\ell=1}^{L} p_{k}(\ell)+P_{c, k}}\right.} & \forall k=1, \ldots, K . \\
\text { s.t. } \quad p_{k}(\ell) \geq 0 \quad \forall \ell=1, \ldots, L, \sum_{\ell=1}^{L} p_{k}(\ell) \leq P_{\max , k} & \forall k=1, \ldots, K
\end{array} .\right.
$$

where we have denoted by $\gamma_{k, a_{k}}^{a s}(\ell)$ the asymptotic expression of the $k$-th user's SINR in the limiting case of $M, K \rightarrow$ $\infty$, with $\frac{K}{M}=\alpha$. We remark that $\gamma_{k, a_{k}}^{a s}(\ell)$ can be evaluated as the limit of the instantaneous SINR (2), instead of the average SINR (5) because the fast-fading channels that appear in (2) will be averaged out in the asymptotic regime. Indeed, recalling that $\boldsymbol{h}_{k, a_{k}}(\ell)=\boldsymbol{R}_{k, a_{k}}^{1 / 2}(\ell) \boldsymbol{\alpha}_{k, a_{k}}(\ell)$, where $\boldsymbol{\alpha}_{k, a_{k}}(\ell)$ is a zero-mean uncorrelated Gaussian vector and that the diagonal entries of $\boldsymbol{R}_{k, a_{k}}^{1 / 2}(\ell)$ have magnitude proportional to $1 / M$, it is possible to leverage [36, Lemma 2.29] to show that $\left\|\boldsymbol{h}_{k, a_{k}}(\ell)\right\|^{2} \rightarrow \operatorname{tr}\left(\boldsymbol{R}_{k, a_{k}}(\ell)\right)$ and $\left|\boldsymbol{h}_{k, a_{k}}(\ell) \boldsymbol{h}_{i, a_{k}}^{H}(\ell)\right|^{2} \rightarrow \operatorname{tr}\left(\boldsymbol{R}_{k, a_{k}}(\ell) \boldsymbol{R}_{i, a_{k}}(\ell)\right)$. Consequently, we have

$$
\begin{aligned}
\gamma_{k, a_{k}}^{a s}(\ell) & =\lim _{\substack{M, K \rightarrow \infty \\
K / M \rightarrow \infty}} \gamma_{k, a_{k}}^{\text {inst }}(\ell) \\
& =\frac{p_{k}(\ell) \operatorname{tr}^{2}\left(\boldsymbol{R}_{k, a_{k}}(\ell)\right)}{\sigma^{2} \operatorname{tr}\left(\boldsymbol{R}_{k, a_{k}}(\ell)\right)+\sum_{i \neq k} p_{i}(\ell) \operatorname{tr}\left(\boldsymbol{R}_{k, a_{k}}(\ell) \boldsymbol{R}_{i, a_{k}}(\ell)\right)},
\end{aligned}
$$

and we notice that, as already anticipated, (35) does not contain the users' channels realizations and therefore can be employed at the transmitters' side for resource allocation purposes.

Now, in the notable case of uncorrelated fading, we have $\boldsymbol{R}_{k, a_{k}}(\ell)=\frac{1}{M} d_{k, a_{k}}^{-\eta} \boldsymbol{I}_{M}$, and (35) becomes

$$
\gamma_{k, a_{k}}^{a s}(\ell)=\frac{p_{k}(\ell) d_{k, a(k)}^{-\eta}}{\sigma^{2}+\frac{1}{M} \sum_{i \neq k} p_{i}(\ell) d_{i, a(k)}^{-\eta}} .
$$

Next, in order to ensure fairness among the users in terms of throughput and delay [5,37], we enforce the constraint that each transmit signal is received with the same power in its assigned receiver, namely $p_{i}(\ell) d_{i, a(i)}^{-\eta}=$ $P_{R}(\ell), \forall i \in \mathcal{L}_{\ell}$, wherein $\mathcal{L}_{\ell}$ is the set of users transmitting on the $\ell$-th carrier. Then, we can write

$$
\gamma_{k, a_{k}}^{a s}(\ell)=\frac{P_{R}(\ell)}{\sigma^{2}+\frac{P_{R}(\ell)}{M} \sum_{i \in \mathcal{L}_{\ell}}\left(\frac{d_{i, a(i)}}{d_{i, a(k)}}\right)^{\eta}} .
$$

Now, in the limit of an infinite number of users $K$, it is reasonable to assume that they will be uniformly distributed over the $L$ available subcarriers, so that each subcarrier is loaded with the same number of users, and the cardinality of the set $\mathcal{L}_{\ell}$ can be taken to be $K / L$. Consequently, the sum at the denominator of (37) converges to $\frac{K}{L} \mu_{k}$, with $\mu_{k}$ being the mean of the random variable defined as the $\eta$-th power of the ratio between the distance of a user randomly located in the network from his assigned receiver, and the distance of the same user from receiver $a_{k}$. Thus, (37) becomes 


$$
\gamma_{k, a_{k}}^{a s}(\ell)=\frac{p_{k}(\ell) d_{k, a(k)}^{-\eta}(\ell)}{\sigma^{2}+\frac{\alpha}{L} \mu_{k} p_{k}(\ell) d_{k, a(k)}^{-\eta}(\ell)},
$$

and we notice that multiuser interference has been averaged out, as it was expected [26]. Equipped with this result, iterations (34) can be recast as

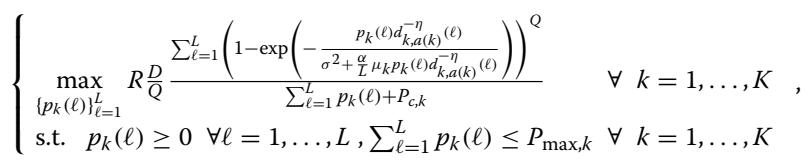

and the following proposition holds.

Proposition 4. Iterations (39) are guaranteed to converge to an equilibrium in $K$ iterations.

Proof. Since multiuser interference is averaged out in the asymptotic SINR, we infer that the $K$ problems in (39) can be decoupled and independently solved. Therefore, after all of the $K$ users have solved their corresponding maximization problem in (39), a fixed point is reached.

We remark that the solution to the generic $k$-th problem in (39) can be obtained by means of the alternating maximization algorithm, in a similar fashion as for Algorithm 2.

\section{Distributed energy-efficient resource allocation in relay-assisted systems}

In this section, we show how the approach proposed in Section 3 extends to two-hop, relay-assisted interference networks. The system model of Section 3 will be modified by adding a fixed, single-antenna, amplify-and-forward (AF) relay between transmitters and receivers. In each symbol interval, the base-band, discrete-time model of the signal received by the relay on subcarrier $\ell$ is given by

$$
y_{r}(\ell)=\sum_{k=1}^{K} \sqrt{p_{k}(\ell)} h_{k}(\ell) b_{k}(\ell)+w_{r}(\ell),
$$

wherein $h_{k}(\ell)$ is the $k$-th user's complex channel coefficient to the relay on subcarrier $\ell$, modeled as a realization of a zero-mean Gaussian random variable with variance $\sigma_{h_{k}}^{2}(\ell)$, while $w_{r}(\ell)$ is the thermal noise on subcarrier $\ell$, modeled as a realization of a zero-mean complex circular Gaussian variable with variance $\sigma_{r}^{2}$. In order to avoid amplifier saturation at the relay, $y_{r}(\ell)$ is to be normalized by the square root of its power $P_{t}(\ell)[25,38]$, which can be computed as

$$
P_{t}(\ell)=E\left[\left|y_{r}(\ell)\right|^{2}\right]=\sigma_{r}^{2}+\sum_{k=1}^{K} p_{k}(\ell)\left|h_{k}(\ell)\right|^{2},
$$

where we have exploited the fact that the noise is uncorrelated with the information symbols, and that information symbols from different users are uncorrelated with each other. After normalization, $y_{r}(\ell)$ is amplified by the amplification factor $a_{R}^{2}(\ell)$ subject to the power constraint $\sum_{\ell=1}^{L} a_{R}^{2}(\ell) \leq P_{r}$, with $P_{r}$ denoting the available relay power. It is to be mentioned that an equivalent approach would be to avoid normalizing $y_{r}(\ell)$ and considering the relay power constraint $\sum_{\ell=1}^{L} a_{R}^{2}(\ell) P_{t}(\ell) \leq P_{r}$. However, such an approach has the drawback that the relay power constraints should also be enforced on the transmitters. Indeed, since the relay has no control over the terms $P_{t}(\ell)$, in order to guarantee that it is always possible to meet the relay power constraint, each transmitter $k$ should allocate its transmit powers $\left\{p_{k}(\ell)\right\}_{\ell=1}^{L}$ not only based on its maximum feasible transmit power but also so as to fulfill the relay power constraint with respect to $\left\{p_{k}(\ell)\right\}_{\ell=1}^{L}$, namely considering the additional constraint

$$
\begin{aligned}
\sum_{\ell=1}^{L} a_{R}^{2}(\ell) p_{k}(\ell)\left|h_{k}(\ell)\right|^{2} \leq & P_{r}-\sum_{\ell=1}^{L} a_{R}^{2}(\ell) \\
& \times\left(\sigma_{r}^{2}+\sum_{i \neq k}^{K} p_{i}(\ell)\left|h_{i}(\ell)\right|^{2}\right)
\end{aligned}
$$

This introduces a coupling between the relay and the mobile users which is not always easy to manage in distributed systems like an interference network. Instead, the normalization approach has the advantage of making the relay completely independent of the mobile users, ensuring that the relay amplifier never goes into saturation without having to enforce additional constraints on the transmitters.

After amplification, the signal is forwarded to the multiple-antenna receivers ${ }^{\mathrm{b}}$. Assuming that the distance between transmitters and receivers is such that the magnitude of the signal received directly from the transmitters is negligible with respect to the relayed signal, the SINR achieved by the $k$-th user in its intended receiver $j=a(k)$ after MRC reception by the filter $h_{k} g_{a_{k}}$ is given by

$$
\gamma_{k, a_{k}}^{\text {inst }}(\ell)=\frac{p_{k}(\ell) a_{R}^{2}(\ell)\left|h_{k}(\ell)\right|^{4}\left\|\boldsymbol{g}_{a_{k}}(\ell)\right\|^{4}}{\sigma^{2}\left|h_{k}(\ell)\right|^{4}\left\|\boldsymbol{g}_{a_{k}}(\ell)\right\|^{2} p_{k}(\ell)+\left|h_{k}(\ell)\right|^{2}\left\|\boldsymbol{g}_{a_{k}}(\ell)\right\|^{2}\left(a_{R}^{2}(\ell)\left\|\boldsymbol{g}_{a_{k}}(\ell)\right\|^{2}+\sigma^{2}\right)\left(\sigma_{r}^{2}+\sum_{i \neq k} p_{i}(\ell)\left|h_{i}(\ell)\right|^{2}\right)}
$$


wherein $\boldsymbol{g}_{j}(\ell)$ denotes the vector channel between the relay and receiver $j$, modeled as a realization of a zeromean Gaussian random vector with covariance matrix $\boldsymbol{R}_{g_{j}}(\ell)$. The main difference of the considered scenario with respect to one-hop systems is that the useful power $p_{k}(\ell)$ also appears at the denominator of the SINR, whereas in the one-hop case the SINR was simply proportional to the useful power.

Now, in order to design a distributed resource allocation algorithm, the first step is to obtain an average expression of the SINR with respect to the users' channels. As for the numerator $N_{k, a_{k}}(\ell)$ of (43), leveraging the results of Section 3.1, we have

$$
\begin{aligned}
E\left[N_{k, a_{k}}(\ell)\right]= & p_{k}(\ell) a_{R}^{2}(\ell) E\left[\left|h_{k}(\ell)\right|^{4}\right] E\left[\left|\boldsymbol{g}_{a_{k}}^{H}(\ell) \boldsymbol{g}_{a_{k}}(\ell)\right|^{2}\right] \\
= & p_{k}(\ell) a_{R}^{2}(\ell) 3 \sigma_{h_{k}}^{4}(\ell)\left(\operatorname{tr}^{2}\left(\boldsymbol{R}_{g_{a_{k}}}(\ell)\right)\right. \\
& \left.+2 \operatorname{tr}\left(\boldsymbol{R}_{g_{a_{k}}}(\ell) \boldsymbol{R}_{g_{a_{k}}}^{H}(\ell)\right)\right)
\end{aligned}
$$

where we have also exploited the fact that $h_{k}(\ell)$ and $\boldsymbol{g}_{a_{k}}(\ell)$ are statistically independent with one another. As for the denominator, the average of the term that depends on the useful power $p_{k}$ can be computed as

$$
p_{k}(\ell) \sigma^{2} E\left[\left|h_{k}(\ell)\right|^{4}\right] E\left[\left\|\boldsymbol{g}_{a_{k}}(\ell)\right\|^{2}\right]=p_{k}(\ell) \sigma^{2} 3 \sigma_{h_{k}}^{4}(\ell) \operatorname{tr}\left(\boldsymbol{R}_{g_{a_{k}}}(\ell)\right),
$$

and the average of the remaining term can be computed similarly as

$$
\begin{aligned}
& E\left[\left|h_{k}(\ell)\right|^{2}\left(a_{R}^{2}(\ell)\left\|\boldsymbol{g}_{a_{k}}(\ell)\right\|^{4}+\sigma^{2}\left\|\boldsymbol{g}_{a_{k}}(\ell)\right\|^{2}\right)\right] \\
& \times E\left[\left(\sigma_{r}^{2}+\sum_{i \neq k} p_{i}(\ell)\left|h_{i}(\ell)\right|^{2}\right)\right]= \\
& \sigma_{h_{k}}^{2}(\ell)\left(a_{R}^{2}(\ell)\left(\operatorname{tr}^{2}\left(\boldsymbol{R}_{g_{a_{k}}}(\ell)\right)+2 \operatorname{tr}\left(\boldsymbol{R}_{g_{a_{k}}}(\ell) \boldsymbol{R}_{g_{a_{k}}}^{H}(\ell)\right)\right)\right. \\
& \left.\quad+\sigma^{2} \operatorname{tr}\left(\boldsymbol{R}_{g_{a_{k}}}(\ell)\right)\right)\left(\sigma_{r}^{2}+\sum_{i \neq k} p_{i}(\ell) \sigma_{h_{i}}^{2}(\ell)\right)
\end{aligned}
$$

Finally, defining for notational convenience the quantities $R_{g_{a_{k}}}(\ell)=\left(\operatorname{tr}^{2}\left(\boldsymbol{R}_{g_{a_{k}}}(\ell)\right)+2 \operatorname{tr}\left(\boldsymbol{R}_{g_{a_{k}}}(\ell) \boldsymbol{R}_{g_{a_{k}}}^{H}(\ell)\right)\right)$ and $z_{k}(\ell)=\sigma_{r}^{2} \sigma_{h_{k}}^{2}(\ell)\left(a_{R}^{2}(\ell) R_{g_{a_{k}}}(\ell)+\sigma^{2} \operatorname{tr}\left(\boldsymbol{R}_{g_{a_{k}}}(\ell)\right)\right)$, for all $k=1, \ldots, K$, the average SINR enjoyed by the $k$-th user in its assigned receiver on subcarrier $\ell$ is expressed as
Next, we follow the approach of Section 3 and consider the optimization problem (6) with (16) as objective function and the new SINR expression (48). The variables to optimize are not only the users' powers but also the relay amplification factors $\left\{a_{R}^{2}(\ell)\right\}_{\ell=1}^{L}$. With respect to the choice of (16) as objective in relay-assisted systems, one remark is in order. The main reason to optimize the EE in wireless networks is to limit the energy consumption of battery-powered terminals, in order to prolong their lifetime. However, fixed relays are usually linked to the electrical supply network and therefore have a virtually indefinite lifetime, which makes the investigation of energy-efficient relaying protocols of little practical interest. For this reason, the power consumed by the relay will not be included in the denominator of (48) and the scenario to be tackled is that in which the relay always employs its full power to maximize (16) in order to help the mobile nodes improve their own energy-saving capabilities. Then, a similar resource allocation algorithm as Algorithm 1 can be stated as follows.

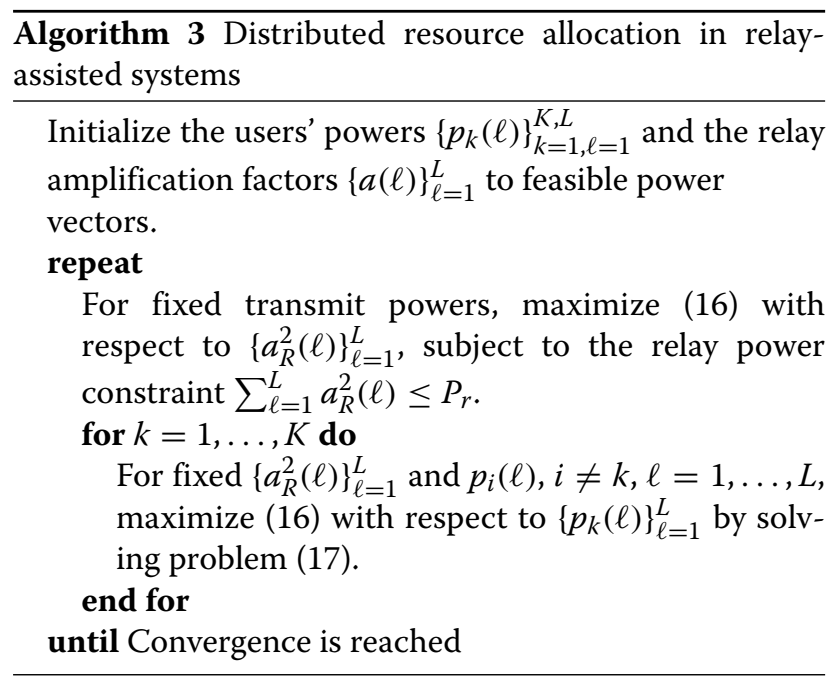

Convergence of Algorithm 3 can be proved with similar arguments as for Algorithm 1, since after each step, the objective (16) is not decreased. In order to implement Algorithm 3, both the relay optimization problem as well as problem (17) with the new SINR expression (48) need to be solved. These two goals are accomplished in the following two sections.

$$
\gamma_{k, a_{k}}(\ell)=\frac{p_{k}(\ell) a_{R}^{2}(\ell) 3 \sigma_{h_{k}}^{4}(\ell) R_{g_{a_{k}}}(\ell)}{p_{k}(\ell) \sigma^{2} 3 \sigma_{h_{k}}^{4}(\ell) \operatorname{tr}\left(\boldsymbol{R}_{g_{a_{k}}}(\ell)\right)+z_{k}(\ell)+\sum_{i \neq k} p_{i}(\ell) \sigma_{h_{i}}^{2}(\ell) \sigma_{h_{k}}^{2}(\ell)\left(\sigma^{2} \operatorname{tr}\left(\boldsymbol{R}_{g_{a_{k}}}(\ell)\right)+a_{R}^{2}(\ell) R_{g_{a_{k}}}(\ell)\right)}
$$




\subsection{Relay optimization}

To begin with, we observe that only the numerator of (16) depends on $\{a(\ell)\}_{\ell=1}^{L}$, through the users' SINRs $\gamma_{k, a_{k}}(\ell)$. Then, the relay optimization problem can be formulated as

$$
\left\{\begin{array}{l}
\max _{\left\{a_{R}^{2}(\ell)\right\}_{\ell=1}^{L}} \prod_{n=1}^{K} \prod_{\ell=1}^{L} e^{-Q \beta_{n} / \gamma_{n, a_{n}}(\ell)} \\
\text { s.t. } \quad a_{R}^{2}(\ell) \geq 0 \quad \forall \ell=1, \ldots, L, \sum_{\ell=1}^{L} a_{R}^{2}(\ell) \leq P_{r}
\end{array} .\right.
$$

The following proposition holds.

Proposition 5. The objective of problem (49) is a log-concave function of $\left\{a_{R}^{2}(\ell)\right\}_{\ell=1}^{L}$.

Proof. Applying the logarithmic function to the objective of (49), we obtain

$$
\ln \prod_{n=1}^{K} \prod_{\ell=1}^{L} e^{-Q \beta_{n} / \gamma_{n, a_{n}}(\ell)}=-Q \sum_{n=1}^{K} \sum_{\ell=1}^{L} \frac{\beta_{n}}{\gamma_{n, a_{n}}(\ell)}=-Q \sum_{n=1}^{K} \sum_{\ell=1}^{L} \frac{\beta_{n}\left(a_{R}^{2}(\ell) \delta_{n}(\ell)+\psi_{n}(\ell)\right)}{a_{R}^{2}(\ell) \phi_{n}(\ell)}
$$

with $\phi_{n}(\ell)=3 p_{n}(\ell) \sigma_{h_{n}}^{4}(\ell) R_{g_{a_{n}}}(\ell), \delta_{n}(\ell)=\sigma_{r}^{2} \sigma_{h_{n}}^{2}(\ell) R_{g_{a_{n}}}(\ell)+\sum_{i \neq n} p_{i}(\ell) \sigma_{h_{n}}^{2}(\ell) \sigma_{h_{i}}^{2}(\ell) R_{g_{a_{n}}}(\ell)$, and $\psi_{n}(\ell)=$ $p_{n}(\ell) \sigma^{2} 3 \sigma_{h_{n}}^{4}(\ell) \operatorname{tr}\left(\boldsymbol{R}_{g_{a_{n}}}(\ell)\right)+\sigma_{r}^{2} \sigma^{2} \sigma_{h_{n}}^{2}(\ell) \operatorname{tr}\left(\boldsymbol{R}_{g_{a_{n}}}(\ell)\right)+\sum_{i \neq n} p_{i}(\ell) \sigma^{2} \sigma_{h_{i}}^{2}(\ell) \sigma_{h_{n}}^{2}(\ell) \operatorname{tr}\left(\boldsymbol{R}_{g_{a_{n}}}(\ell)\right)$. Denote by $v_{n}(\ell)$ the generic summand in (50). Computing the second-order derivative of $v_{n}(\ell)$ with respect to $a_{R}^{2}(\ell)$ yields

$$
\frac{\partial^{2} v_{n}(\ell)}{\partial^{2} a_{R}^{2}(\ell)}=-\frac{2 Q \beta_{n} \psi_{n}(\ell)}{\phi_{n}(\ell)\left(a_{R}^{2}(\ell)\right)^{3}} \leq 0
$$

As a consequence, the relay amplification factors on each subcarrier can be found by solving a convex problem equivalent to (49), which is obtained by simply considering the logarithm of the objective.

\subsection{Transmitters optimization}

In order to solve problem (17) for all $k=1, \ldots, K$, the following proposition is provided, which extends the results of Proposition 3 to the relay-assisted scenario.

Proposition 6. Assume the generic $k$-th user's average SINR is expressed as in (48). Then, for any $k=1, \ldots, K$, problem (17) can be restated as

$$
\left\{\begin{array}{l}
\max _{\left\{p_{k}(\ell)\right\}_{\ell=1}^{L}}-\sum_{\ell=1}^{L}\left(\frac{p_{k}(\ell) \mu_{k}(\ell)+v_{k}(\ell)}{p_{k}(\ell) \alpha_{k}(\ell)}+\lambda_{k}(\ell) p_{k}(\ell)+\ln \left(p_{k}(\ell)+P_{c, k}\right)\right) \\
\text { s.t. } \quad p_{k}(\ell) \geq 0 \quad \forall \ell=1, \ldots, L, \sum_{\ell=1}^{L} p_{k}(\ell) \leq P_{\max , k}
\end{array} .\right.
$$

wherein

$$
\begin{aligned}
\mu_{k}(\ell) & =Q \beta_{k} \sigma^{2} 3 \sigma_{h_{k}}^{4}(\ell) \operatorname{tr}\left(\boldsymbol{R}_{g_{a_{k}}}(\ell)\right), v_{k}(\ell) \\
& =Q \beta_{k}\left(z_{k}(\ell)+\sum_{i \neq k} p_{i}(\ell) \sigma_{h_{k}}^{2}(\ell) \sigma_{h_{i}}^{2}(\ell)\left(\sigma^{2} \operatorname{tr}\left(\boldsymbol{R}_{g_{a_{k}}}(\ell)\right)+a_{R}^{2}(\ell) R_{g_{a_{k}}}(\ell)\right)\right) \\
\alpha_{k}(\ell) & =3 a_{R}^{2}(\ell) \sigma_{h_{k}}^{4}(\ell) R_{g_{a_{k}}}(\ell), \lambda_{k}(\ell) \\
& =Q \sum_{n \neq k} \beta_{n} \frac{\sigma^{2} \sigma_{h_{k}}^{2}(\ell) \sigma_{h_{n}}^{2}(\ell) \operatorname{tr}\left(\boldsymbol{R}_{g_{a_{n}}}(\ell)\right)+a_{R}^{2}(\ell) \sigma_{h_{n}}^{2}(\ell) \sigma_{h_{k}}^{2}(\ell) R_{g_{a_{n}}}(\ell)}{3 a_{R}^{2}(\ell) \sigma_{h_{n}}^{4}(\ell) R_{g_{a_{n}}}(\ell) p_{n}(\ell)} .
\end{aligned}
$$

Moreover, the objective of (52) has a unique maximizer, which lies in its concave region. 
Proof. The proof of this result follows along the same lines as the proof of Proposition 3. First, applying the logarithmic function to (16), we obtain

$$
K \ln \left(\frac{R D}{Q}\right)+\ln \left(\prod_{n=1}^{K} \prod_{\ell=1}^{L} \frac{\left(e^{-Q \beta_{n} / \gamma_{n, a_{n}}(\ell)}\right)}{\left(p_{n}(\ell)+P_{c, n}\right)}\right)=K \ln \left(\frac{R D}{Q}\right)-\sum_{n=1}^{K} \sum_{\ell=1}^{L}\left(\frac{Q \beta_{n}}{\gamma_{n, a_{n}}(\ell)}+\ln \left(p_{n}(\ell)+P_{c, n}\right)\right) .
$$

Next, plugging the average SINR expression (48) into (55) yields

$$
\begin{aligned}
& -Q \beta_{k} \sum_{\ell=1}^{L} \frac{p_{k}(\ell) \sigma^{2} 3 \sigma_{h_{k}}^{4}(\ell) \operatorname{tr}\left(\boldsymbol{R}_{g_{a_{k}}}(\ell)\right)+z_{k}(\ell)+\sum_{i \neq k} p_{i}(\ell) \sigma_{h_{k}}^{2}(\ell) \sigma_{h_{i}}^{2}(\ell)\left(\sigma^{2} \operatorname{tr}\left(\boldsymbol{R}_{g_{a_{k}}}(\ell)\right)+a_{R}^{2}(\ell) R_{g_{a_{k}}}(\ell)\right)}{p_{k}(\ell) 3 a_{R}^{2}(\ell) \sigma_{h_{k}}^{4}(\ell) R_{g_{a_{k}}}(\ell)} \\
& -\sum_{n \neq k} \sum_{\ell=1}^{L} p_{k}(\ell) Q \beta_{n} \frac{\sigma^{2} \sigma_{h_{k}}^{2}(\ell) \sigma_{h_{n}}^{2}(\ell) \operatorname{tr}\left(\boldsymbol{R}_{g_{a_{n}}}(\ell)\right)+a_{R}^{2}(\ell) \sigma_{h_{n}}^{2}(\ell) \sigma_{h_{k}}^{2}(\ell) R_{g_{a_{n}}}(\ell)}{p_{n}(\ell) 3 a_{R}^{2}(\ell) \sigma_{h_{n}}^{4}(\ell) R_{g_{a_{n}}}(\ell)} \\
& -\sum_{\ell=1}^{L} \ln \left(p_{k}(\ell)+P_{c, k}\right)+C_{k},
\end{aligned}
$$

with $C_{k}$ including all the terms that do not depend on the $k$-th user's transmit powers. Then, it can be seen that (56) coincides with the objective of (52), which proves the first part of the thesis. As for the second part, let us denote each summand of the objective of (18) as the function

$$
f_{k}\left(p_{k}(\ell)\right)=-\left(\frac{p_{k}(\ell) \mu_{k}(\ell)+v_{k}(\ell)}{p_{k}(\ell) \alpha_{k}(\ell)}+\lambda_{k}(\ell) p_{k}(\ell)+\ln \left(p_{k}(\ell)+P_{c, k}\right)\right) .
$$

Next, we note that $f_{k}\left(p_{k}(\ell)\right)$ can be equivalently written as

$$
f_{k}\left(p_{k}(\ell)\right)=-\left(\frac{\mu_{k}(\ell)}{\alpha_{k}(\ell)}+\frac{v_{k}(\ell) / \alpha_{k}(\ell)}{p_{k}(\ell)}+\lambda_{k}(\ell) p_{k}(\ell)+\ln \left(p_{k}(\ell)+P_{c, k}\right)\right) \text {. }
$$

Comparing (58) with (23), it follows that, up to the constant term $\frac{\mu_{k}(\ell)}{\alpha_{k}(\ell)}$, for all $\ell=1, \ldots, L, f_{k}\left(p_{k}(\ell)\right)$ is formally equivalent to the function $g_{k}\left(p_{k}(\ell)\right)$ given by (23), which was encountered in the proof of Proposition 3. Consequently, since the constant term $\frac{\mu_{k}(\ell)}{\alpha_{k}(\ell)}$ vanishes when computing the derivatives of (58), and since the properties that were shown to hold for $g_{k}\left(p_{k}(\ell)\right)$ in the proof of Proposition 3 were derived assuming no particular expression for the coefficients $a_{k}(\ell)$ and $c_{k}(\ell)$, the two functions $f_{k}\left(p_{k}(\ell)\right)$ and $g_{k}\left(p_{k}(\ell)\right)$ will enjoy similar properties and the line of reasoning employed in Proposition 3 can be replicated here to obtain the thesis.

Therefore, similarly to the one-hop case, also in the relay-assisted scenario, it is possible to recast the generic $k$-th problem in (6) in convex form without loss of generality by simply restricting the feasible set to the concave region of the objective. Moreover, for applications in which computational complexity is a major issue, a similar algorithm as Algorithm 2 can be devised resorting to the alternating maximization technique, in order to convert problem (17) into a sequence of scalar problems.

\section{Numerical results}

An interference network operating in a square area of $2,800 \times 2,800 \mathrm{~m}^{2}$ has been considered. The network consists of $J=4$ receivers, each one equipped with $M=4$ antennas, placed at points inside the square with coordinates (700; 700), (700;2, 100), (2, 100; 700), and $(2,100 ; 2,100)$, respectively, and of mobile users placed randomly inside the square. Each user is assigned to the receiver toward which he has the best channel coefficient. The system bit rate has been set to $R=100 \mathrm{kbits} / \mathrm{s}$, the maximum feasible transmit power to $P_{\max , k}=0$ $\mathrm{dBW}$ for all $k=1, \ldots, K$, the noise power to $\sigma^{2}=$ $10^{-9} \mathrm{~W}$, the power decay factor to $\eta=2$, and the circuit power $P_{c, k}=100 \mathrm{dBm}$, for all $k=1, \ldots, K$. The performance is analyzed with reference to the transmission of each single symbol, thus setting $Q=1$. The channel vectors $\boldsymbol{h}_{k, a_{k}}(\ell)$ have been modeled as zero-mean Gaussian random vectors with covariance matrix $\frac{d_{k, a_{k}}^{-\eta}}{M} \boldsymbol{R}$, where $\boldsymbol{R}$ has been generated according to the exponential correlation model with correlation coefficient $\rho$. The presented results have been obtained by averaging over $10^{4}$ random realizations for all system parameters. The number of active users ranges up to $K=15$, while the number of subcarriers available to each user has been set to $L=3$. Thus, a very hostile operating condition is considered, since the network load ranges up to $K / L=5$. Figures 2 and 3 and Table 1 refer to the case $\rho=0$, thus addressing the notable case of independent channel coefficients. Figures 2 and 3 address the performance 


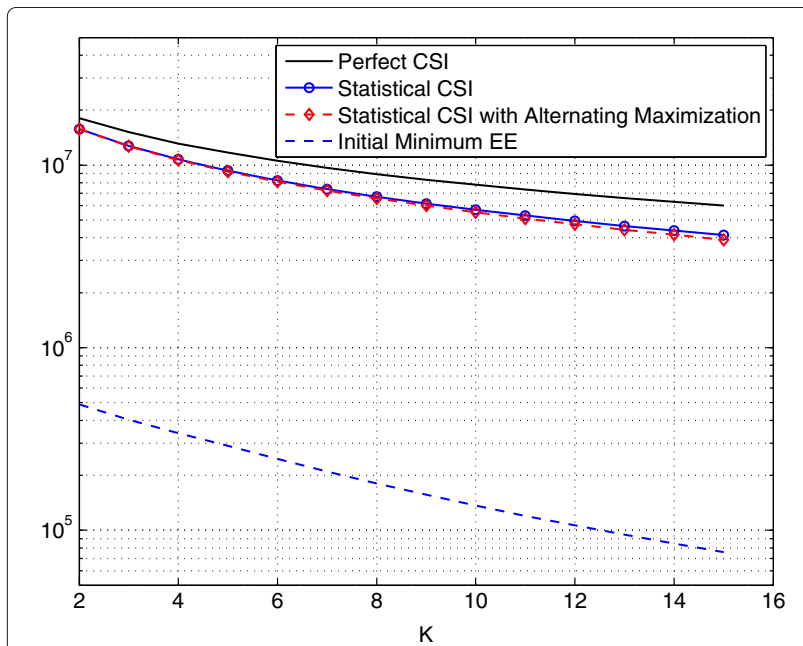

Figure $\mathbf{2} \boldsymbol{\rho}=\mathbf{0}$. Minimum EE [bits/J]. (blue solid line with circle) Fixed point of Algorithm 1 with alternating maximization; (red dashed line with diamond) fixed point of Algorithm 1 with convex reformulation of problem (18); (black solid line) Algorithm 1 implemented with instantaneous, perfect CSI; (blue dashed line) initial minimum EE.

of Algorithm 1. Figure 2 evaluates the performance of Algorithm 1 in terms of the minimum EE, over the users, versus the number of active users. Otherwise stated, the shown performance metric is $\min _{k} \mathrm{EE}_{k}$, with $\mathrm{EE}_{k}$ being the standard EE (8), achieved at the fixed point of Algorithm 1. It is to be stressed that the instantaneous EE has been plotted, meaning that once Algorithm 1 has converged, the resulting transmit powers have been used to evaluate the instantaneous achieved SINR (2) and then to

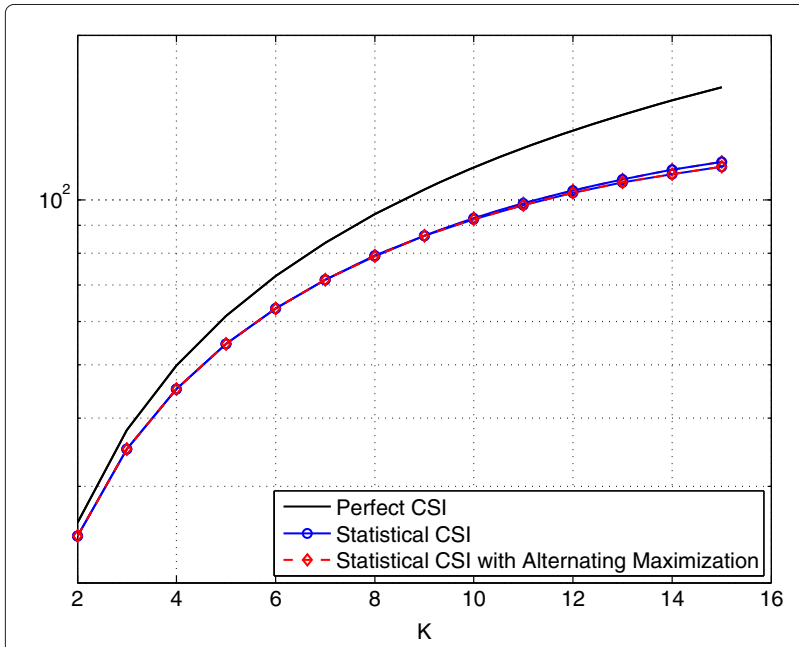

Figure $3 \boldsymbol{\rho}=\mathbf{0}$. Achieved value of (21). (Blue solid line with circle) Fixed point of Algorithm 1 with alternating maximization; (red dashed line with diamond) fixed point of Algorithm 1 with convex reformulation of problem (18); (black solid line) fixed point of Algorithm 1 implemented with instantaneous, perfect CSI.
Table $1 \rho=0$, average number of outer loops needed for Algorithm 1 to converge versus the number of active users

\begin{tabular}{lcc}
\hline & Instantaneous CSI & Statistical CSI \\
\hline$K=2$ & 4.90 & 4.25 \\
$K=4$ & 7.59 & 6.25 \\
\hline$K=6$ & 9.17 & 7.39 \\
\hline$K=8$ & 10.49 & 8.39 \\
\hline$K=10$ & 11.54 & 9.29 \\
\hline$K=12$ & 12.63 & 10.05 \\
\hline$K=14$ & 13.85 & 10.80
\end{tabular}

Convergence is declared when $E_{\ell}^{(i)}=\frac{\left\|\boldsymbol{p}^{(i)}(\ell)-\boldsymbol{p}^{(i-1)}(\ell)\right\|^{2}}{\left\|\boldsymbol{p}^{(i)}(\ell)\right\|^{2}} \leq 10^{-6}$, for all $\ell=1, \ldots, L$, with $\boldsymbol{p}^{(i)}(\ell)=\left\{p_{k}^{(i)}(\ell)\right\}_{k=1}^{K}$ being the power vector after the $i$-th outer loop.

compute the EE (8). The following scenarios have been contrasted:

- Algorithm 1 implemented by solving the generic $k$-th problem of (17) according to Algorithm 2

- Algorithm 1 implemented by solving the generic $k$-th problem of (17) by means of its convex reformulation.

- As a benchmark, the minimum EE obtained when Algorithm 1 is run with perfect, instantaneous CSI is reported.

- Initial minimum EE resulting from a random power allocation over the available subcarriers. This scenario is shown for comparison purposes, since it represents the minimum EE before the resource allocation scheme comes into play.

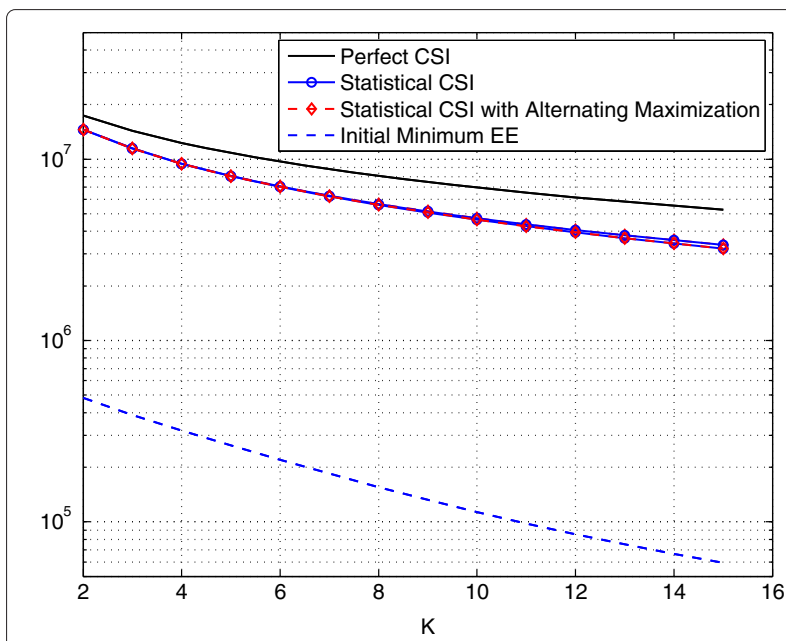

Figure $4 \boldsymbol{\rho}=\mathbf{0 . 5}$, minimum EE [bits/J]. (Blue solid line with circle) Fixed point of Algorithm 1 with alternating maximization; (red dashed line with diamond) fixed point of Algorithm 1 with convex reformulation of problem (18); (black solid line) Algorithm 1 implemented with instantaneous, perfect CSI; (blue dashed line) initial minimum EE. 


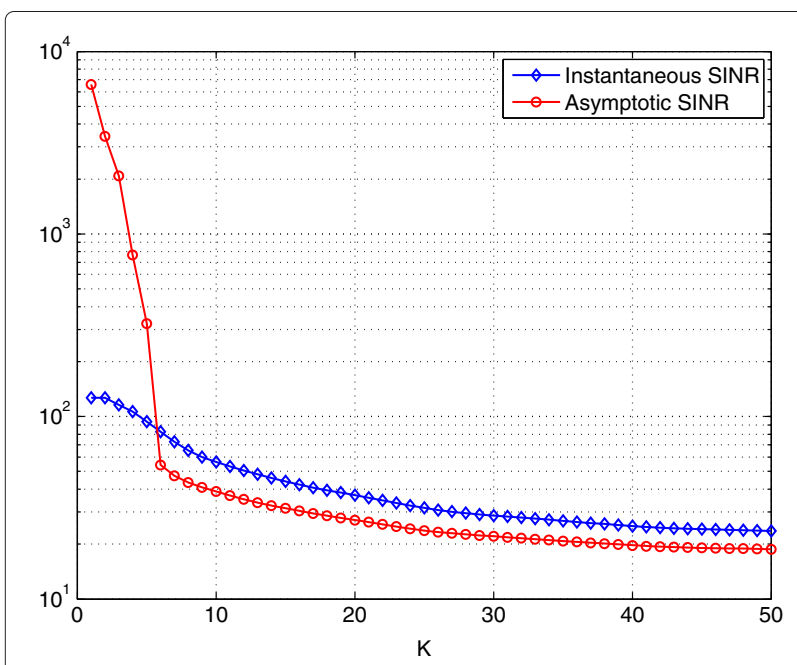

Figure 5 Average instantaneous SINR against its asymptotic approximation. $\alpha=0.5$.

Results clearly show that the proposed games with statistical CSI largely improve the initial EE while suffering a limited gap with respect to the instantaneous CSI benchmark, even for very high network loads. It is also interesting to note how the performance of Algorithm 1 are virtually identical when problem (17) is solved by means of Algorithm 2 and by its convex reformulation, thus indicating that the low-complexity Algorithm 2 can effectively substitute standard numerical algorithms.

Figure 3 considers a similar scenario, with the difference that the shown performance metric is the social welfare function (21). Similar remarks as for Figure 2 hold.

Table 1 contrasts the average number of outer loops needed for Algorithm 1 to reach convergence when statistical and instantaneous CSI is available. As expected, the number of iterations increases with the network load, but it is still satisfactory even for high network loads. Also, it is seen that the number of required iterations with statistical CSI is only slightly lower than that with perfect CSI.

Next, we focus on the case in which the network is affected by correlated fading. In Figure 4, it has been set $\rho=0.5$, and a similar scenario as in Figure 2 is shown. It is seen that also with correlated fading, Algorithm 1 enjoys similar properties as with uncorrelated fading, and similar remarks as for the case $\rho=0$ can be made. Similar results, which are omitted for brevity's sake, have been obtained when the performance metric is the social welfare function (21) instead of the minimum EE.

Finally, in Figure 5, the asymptotic scenario of Section 4 is addressed. In order to obtain an insight as to how large $K$ and $M$ need to be for the asymptotic expression (36) to be valid, the average, over the users and subcarriers, instantaneous SINR $\frac{1}{K L} \sum_{k=1}^{K} \sum_{\ell=1}^{L} \gamma_{k, a_{k}}^{i n s}(\ell)$ and its asymptotic approximation $\frac{1}{K L} \sum_{k=1}^{K} \sum_{\ell=1}^{L} \gamma_{k, a_{k}}^{a s}(\ell)$, with $\gamma_{k, a_{k}}^{a s}(\ell)$ expressed as in (36), have been contrasted for increasing $K$ and $M=K / \alpha$, with $\alpha=0.5$. The results show that a good approximation is already obtained for $K \approx 20$.

\section{Conclusions}

This paper has dealt with the problem of distributed resource allocation in the uplink of a multi-carrier interference network with multiple receive antennas. A performance metric that trades off between the need for improved overall EE and the need for a fair resource allocation has been proposed, and distributed resource allocation algorithms that are guaranteed to converge to a stable equilibrium have been devised, relying only on statistical CSI at the transmitters' side. Both one-hop and two-hop networks have been considered. The proposed algorithms have an affordable computational load, since they only require the solution of convex problems. Individual EE maximization in one-hop saturated network in which the number of users and of receive antennas in each receiver grow large with a fixed ratio has also been tackled. Also in this case, a distributed resource allocation algorithm that converges to a stable equilibrium has been designed. Finally, numerical results have been provided to assess the performance of the proposed procedures.

\section{Endnotes}

${ }^{\mathrm{a}} \mathrm{A}$ function is said to be $\mathrm{S}$-shaped if there exists a point below which it is convex and above which it is concave.

${ }^{\mathrm{b}}$ The forwarded signal is transmitted on an orthogonal channel, usually by means of frequency or time division duplex, to avoid interference with the incoming signal from the users.

\section{Competing interests}

The authors declare that they have no competing interests.

\section{Acknowledgements}

The work of Alessio Zappone has received funding from the German Research Foundation (DFG) project CEMRIN, under grant ZA 747/1-1. The work of Giuseppa Alfano, Stefano Buzzi, and Michela Meo has received funding from the European Union Seventh Framework Programme (FP7/2007-2013) under grant agreement no. 257740 (Network of Excellence "TREND"), from the Regional Project MASP, Regione Piemonte, Torinowireless 2009, and by the Network of Excellence NEWCOM $\sharp$, grant agreement no. 318306.

\section{Author details}

${ }^{1}$ Dresden University of Technology, Communication Theory Laboratory, Dresden 01062, Germany. ${ }^{2}$ Dipartimento di Elettronica e Telecomunicazioni, Politecnico di Torino, Corso Duca degli Abruzzi 24, Torino 10129, Italy.

${ }^{3}$ Consorzio Nazionale Interuniversitario per le Telecomunicazioni (CNIT) and University of Cassino and Lazio Meridionale, DIEl, Via G. Di Biasio 43, Cassino (FR) 03043, Italy.

Received: 19 December 2012 Accepted: 22 July 2013 Published: 9 August 2013

\section{References}

1. D Goodman, N Mandayam, Power control for wireless data. IEEE Pers. Commun. 7, 48-54 (2000) 
2. C Saraydar, N Mandayam, D Goodman, Efficient power control via pricing in wireless data networks. IEEE Trans. Commun. 50(2), 291-303 (2002)

3. CU Saraydar, NB Mandayam, DJ Goodman, Pricing and power control in a multicell wireless data network. IEEE J. Select. Areas Commun. 19(10), 1883-1892 (2001)

4. F Meshkati, HV Poor, SC Schwartz, NB Mandayam, An energy-efficient approach to power control and receiver design in wireless data networks. IEEE Trans. Commun. 53(11), 1885-1894 (2005)

5. S Buzzi, HV Poor, Joint receiver and transmitter optimization for energy-efficient CDMA communications. IEEE J. Select. Areas Commun. 26(3), 459-472 (2008)

6. S Buzzi, H Poor, A Zappone, Transmitter waveform and widely linear receiver design: noncooperative games for wireless multiple-access networks. IEEE Trans. Inf. Theory 56(10), 4874-4892 (2010)

7. S Lasaulce, Y Hayel, R El Azouzi, M Debbah, Introducing hierarchy in energy games. IEEE Trans. Wireless Commun. 8(7), 3833-3843 (2009)

8. M Treust, $\mathrm{S}$ Lasaulce, A repeated game formulation of energy-efficient decentralized power control. IEEE Trans. Wireless Commun. 9(9), 2860-2869 (2010)

9. A Zappone, S Buzzi, E Jorswieck, Energy-efficient power control and receiver design in relay-assisted DS/CDMA wireless networks via game theory. IEEE Commun. Lett. 15(7), 701-703 (2011)

10. F Meshkati, M Chiang, H Poor, S Schwartz, A game-theoretic approach to energy-efficient power control in multicarrier CDMA systems. IEEE J. Select. Areas Commun. 24(6), 1115-1129 (2006)

11. M Moretti, A Todini, A resource allocator for the uplink of multi-cell OFDMA systems. IEEE Trans. Wireless Commun. 6(8), 2807-2812 (2007)

12. Z Han, Z Ji, K Liu, Non-cooperative resource competition game by virtual referee in multi-cell OFDMA networks. IEEE J. Select. Areas Commun. 25(6), 1079-1090 (2007)

13. K Yang, N Prasad, $X$ Wang, An auction approach to resource allocation in uplink OFDMA systems. IEEE Trans. Signal Proc. 57(11), 4482-4496 (2009)

14. G Bacci, A Bulzomato, M Luise, in Int. Conf. on Performance Evaluation Methodologies and Tools (ValueTools). Uplink power control and subcarrier assignment for an OFDMA multicellular network based on game theory. (Paris, 16-20 May 2011)

15. S Buzzi, G Colavolpe, D Saturnino, A Zappone, Potential games for energyefficient power control and subcarrier allocation in uplink multicell OFDMA systems. IEEE J. Select. Topics Signal Proc. 6(2), 89-103 (2012)

16. D Monderer, L Shapley, Potential games. Games Econ. Behav.

14, 124-143 (1996)

17. G Scutari, S Barbarossa, D Palomar, in 2006 IEEE Internationa Conference on Acoustics, Speech and Signal Processing. Potential games: a framework for vector power control problems with coupled constraints. (Toulouse, 14)

18. DL Quang, HC Yong, BH Soong, An interference-minimization potential game for OFDMA-based distributed spectrum sharing systems. IEEE Trans. Vehic. Tech. 60(7), 3374-3385 (2011)

19. DL Quang, HC Yong, BH Soong, Performance analysis of downlink multi-cell OFDMA systems based on potential game. IEEE Trans. Wireless Commun. 11(9), 3358-3367 (2012)

20. S Betz, HV Poor, Energy efficient communications in CDMA networks: a game theoretic analysis considering operating costs. IEEE Trans. Signal Proc. 56(10), 5181-5190 (2008)

21. G Miao, N Himayat, GY Li, S Talwar, Distributed interference-aware energy-efficient power optimization. IEEE Trans. Wireless Commun. 10(4), 1323-1333 (2011)

22. Z Chong, EA Jorswieck, in IEEE Online Green Communications Conference. Energy-efficient power control for MIMO time-varying channels. (26-29 September 2011)

23. C Isheden, Z Chong, E Jorswieck, G Fettweis, Framework for link-level energy efficiency optimization with informed transmitter. IEEE Trans. Wireless Commun. 11(8), 2946-2957 (2012)

24. A Zappone, Z Chong, E Jorswieck, S Buzzi, in Third International Conference on Future Energy Systems (E-energy 2012). Green resource allocation in relay-assisted multicarrier IC networks considering circuit dissipated power. (Madrid, 9)

25. A Zappone, Z Chong, E Jorswieck, S Buzzi, Energy-aware competitive power control in relay-assisted interference wireless networks. IEEE Trans. Wireless Commun. 12(4), 1860-1871 (2013)
26. T Marzetta, Noncooperative cellular wireless with unlimited numbers of base station antennas. IEEE Trans. Wireless Commun. 9(11), 3590-3600 (2010)

27. N Jindal, J Andrews, S Weber, Multi-antenna communication in ad hoc networks: Achieving MIMO gains with SIMO transmission. IEEE Trans. Commun. 59(2), 529-540 (2011)

28. H Huh, G Caire, H Papadopoulos, S Ramprashad, Achieving massive MIMO spectral efficiency with a not-so-large number of antennas. [http://arxiv. org/abs/1107.3862] Accessed 13 September 2011

29. E Belmega, S Lasaulce, Energy-efficient precoding for multiple-antenna terminals. IEEE Trans. Signal Proc. 59, 329-340 (2011)

30. F Meshkati, A Goldsmith, H Poor, S Schwartz, A game-theoretic approach to energy-efficient modulation in CDMA networks with delay QoS constraints. IEEE J Select. Areas Commun. 25(6), 1069-1078 (2007)

31. A Ghosh, J Zhang, JG Andrews, R Muhamed, Fundamentals of LTE (Prentice Hall Communications Engineering and Emerging Technologies Series, Upper Saddle River, NJ, 2011)

32. DWK Ng, ES Lo, R Schober, Energy-efficient resource allocation in multi-cell OFDMA systems with limited Backhaul capacity. IEEE Trans. Wireless Commun. 11(10), 3618-3631 (2012)

33. R Mazumdar, LG Mason, C Dougligeris, Fairness in network optimal flow control: optimality of product forms. IEEE Trans. Commun. 39(5), 775-782 (1991)

34. H Boche, S Naik, Revisiting proportional fairness: anonymity among users in interference coupled wireless systems. IEEE Trans. Commun. 58(10), 2995-3000 (2010)

35. D Bertsekas, Nonlinear Programming, 2nd edn. (Athena Scientific, Belmont, MA, 1999)

36. A Tulino, S Verdú, Random matrix theory and wireless communications, vol. 1 (Now Publishers Inc, Hanover, MA, 2004)

37. D Tse, S Hanly, Linear multiuser receivers: Effective interference, effective bandwidth and user capacity. IEEE Trans. Inf Theory 45, 641-657 (1999)

38. S Ren, M van der Schaar, Pricing and distributed power control in wireless relay networks. IEEE Trans. Signal Proc. 59(6), 2913-2926 (2011)

doi:10.1186/1687-1499-2013-205

Cite this article as: Zappone et al:: Distributed energy-aware resource allocation in multi-antenna multi-carrier interference networks with statistical CSI. EURASIP Journal on Wireless Communications and Networking 2013 2013:205.

\section{Submit your manuscript to a SpringerOpen ${ }^{\circ}$ journal and benefit from:}

- Convenient online submission

- Rigorous peer review

- Immediate publication on acceptance

- Open access: articles freely available online

- High visibility within the field

- Retaining the copyright to your article

Submit your next manuscript at $>$ springeropen.com 